NBER WORKING PAPER SERIES

\title{
PHARMACEUTICAL USE FOLLOWING GENERIC ENTRY: PAYING LESS AND BUYING LESS
}

Peter J. Huckfeldt

Christopher R. Knittel

Working Paper 17046

http://www.nber.org/papers/w17046

\author{
NATIONAL BUREAU OF ECONOMIC RESEARCH \\ 1050 Massachusetts Avenue \\ Cambridge, MA 02138
}

May 2011

This paper has benefited substantially from conversations with Hilary Hoynes, Doug Miller, Marianne Page, Ann Huff Stevens, and seminar participants at UC Davis and UC Berkeley. Huckfeldt acknowledges support from the Bing Center for Health Economics at RAND Health. All remaining errors are our

own. The views expressed herein are those of the authors and do not necessarily reflect the views of the National Bureau of Economic Research.

NBER working papers are circulated for discussion and comment purposes. They have not been peerreviewed or been subject to the review by the NBER Board of Directors that accompanies official NBER publications.

(C) 2011 by Peter J. Huckfeldt and Christopher R. Knittel. All rights reserved. Short sections of text, not to exceed two paragraphs, may be quoted without explicit permission provided that full credit, including $(\odot)$ notice, is given to the source. 
Pharmaceutical Use Following Generic Entry: Paying Less and Buying Less

Peter J. Huckfeldt and Christopher R. Knittel

NBER Working Paper No. 17046

May 2011

JEL No. I18,L11,M3,O31,O38

\begin{abstract}
$\underline{\text { ABSTRACT }}$
We study the effects of generic entry on prices and utilization using both event study models that exploit the differential timing of generic entry across drug molecules and cast studies. Our analysis examines drugs treating hypertension, high blood pressure, type 2 diabetes, and depression using price and utilization data from the Medical Expenditure Panel Survey. We find that utilization of drug molecules starts decreasing in the two years prior to generic entry and continues to decrease in the years following generic entry, despite decreases in prices offered by generic versions of a drug. This decrease coincides with the market entry and increased utilization of branded reformulations of a drug going off patent. We show case study evidence that utilization patterns coincide with changes in marketing by branded drug manufacturers. While the reformulations---often extended-release versions of the patent-expiring drug---offer potential health benefits, the FDA does not require evidence that the reformulations are improvements over the previous drug in order to grant a patent. Indeed, in a number of experiments comparing the efficacies of the patent-expiring and reformulated drugs do not find statistical differences in health outcomes calling into question the patent-extension policy.
\end{abstract}

\author{
Peter J. Huckfeldt \\ RAND Corporation \\ 1776 Main Street, P.O. Box 2138 \\ Santa Monica, CA 90407-2138 \\ huckfeld@rand.org \\ Christopher R. Knittel \\ MIT Sloan School of Management \\ 100 Main Street, E62-513 \\ Cambridge, MA 02142 \\ and NBER \\ knittel@mit.edu
}


Over the next few years, a number of blockbuster drugs face patent expirationt is estimated that by 2012, brands with more than $\$ 30$ billion in sales will face new competition from generics. As more brands face patent expiration, many manufacturers will face the dilemma of how to grow revenue and minimize operational cutbacks as reliance on the new drug pipeline is unrealistic. One tactic is to develop an extended release formulation of an existing brand. Whether you call it extended release (ER, XR), long-acting (LA), or extra-long (XL), the modified formulation is intended to simplify dosing, improve compliance and extend the life of the patent. (emphasis added)

Kelly Renfro, Marketing Manager for McKesson Patient Relationship Solutions $\mathbb{1}^{1}$

\section{Introduction}

Among policymakers, the prescription drug industry is often touted as being a major source of inefficiency of the US health care system. This is not surprising given that annual expenditures on prescription drugs exceeded $\$ 234$ billion in 2008, accounting for over 1.5 percent of US GDP (Lundy, 2010). The growth in expenditures has been dramatic; expenditures were less than $\$ 25$ billion in 1970 (in 2008 dollars) $2^{2}$ These increases come despite increases in the share of prescriptions filled with generic drugs. In 1984, only 19 percent of prescriptions were generics; this was 67 percent in $2 0 0 7 \longdiv { 3 }$

The rise in generics is due in large part to the Hatch-Waxman Act of 1984 which considerably lowered the fixed costs associated with generic entry. Following this act, a branded drug often faces a large amount of competition soon after patent expiration. We use the discontinuity in the amount of competition to analyze how pricing and prescription behavior varies around generic entry. Our empirical strategy uses utilization and price data from the Medical Expenditure Panel Survey, advertising data from IMS Health, and a mix of case studies and event study models to estimate the effects of generic entry on total and out-of-pocket prices; brand, generic, and total drug molecule utilization; and the utilization of branded reformulations of a drug facing generic competition 4

\footnotetext{
${ }^{1}$ http://www.pharmaphorum.com/2011/03/18/battling-patent-expiration-by-building-brand-loyalty/

${ }^{2}$ See Schondelmeyer and Thomas (1990).

${ }^{3}$ The 1984 figure is taken from CBO $(1998)$ and is the share of countable units, while the 2007 number is from Hartman et al. (2009a) and is the share of prescriptions.

${ }^{4}$ Throughout the paper we refer to a drug, or molecule, drug molecule, or product interchangeably. By this, we are referring to a distinct "molecular entity". For example, metformin and metformin extended release are two distinctive molecules, with brand and generic versions.
} 
Our focus is on therapeutic classes that treat depression, hypertension, high cholesterol, and type 2 diabetes from 1996 and 2007.

We find decreases in pharmacy and patient out-of-pocket prices for the molecule following generic entry, and accompanying decreases in brand market shares. Two years after entry, total prices of the generic version of a molecule are 5-10 percent below pre-entry prices, while patients' out-of-pocket expenditures are 15 percent lower. However, we find large decreases in overall use after patent expiration that begin in the two years before generic entry and continue in the years following. From two years prior to entry to two years after entry utilization falls, on average, by almost 50 percent. We also find that the decrease in overall utilization prior to generic entry coincides with increased utilization of branded reformulations of molecules going off patent. We find similar patterns of utilization across different types of health plans.

What explains the decrease in both molecular prices and utilization? We show evidence from a number of case studies suggesting that the link is advertising. Specifically, we show that advertising decreases substantially in the two years prior to generic competition, coinciding with increased advertising for patented reformulations, typically extended-release versions of the original molecule. Thus, advertising effectively shifts demand from the now cheaper original molecule to other, stillpatented, molecules.

The welfare consequences of this are ambiguous and hinge on the relative benefits of extendedrelease versions and the inefficiency arising from advertising and market power. These issues are cleanly summarized in our opening quote. While extended-release versions are likely to increase patient compliance and tolerability of the medication, these potential benefits need to be weighed against the distortions from market power. It is also worth noting that the benefits from extendedrelease versions are not always clear. Regulatory approval for an extended-release version does not require proof that the extended-release version is an improvement over the immediate-release version. The medical literature has pointed to one potential downside of extended-release versions is the increase the risks associated with missing a dose, leading some to question their value over immediate-release versions $5^{5}$ There are few studies comparing the efficacy of extended-release versions with their immediate-release counterpart, rather than a placebo. Those that do exist show a mixture of potential benefits (see, for example, Rudolph and Feiger (1999) and Swanson et al. (2004)) and no statistically (or medically) significant differences (see, for example, Adler et al.

\footnotetext{
${ }^{5}$ See, for example, Perucca (2009).
} 
(2000), Dopfner et al. (2004), and Schwartz et al. (April 2006)). Furthermore, for some classes of drugs such as painkillers, extended-release versions create a heightened risk of abuse because a larger quantity of the drug is contained in one pill. Famous cases of abuse, for example issues with Oxycontin - the extended-release version of OxyCodone, likely prompted the FDA to recently require a Risk Evaluation and Mitigation Strategy (REMS) for extended-release opioids.

We are not the first to analyze how prices, quantities, and promotional activities are affected by patent expiration in the pharmaceutical industry. The analyses of Caves et al. (1991), Berndt et al. (2003), Huskamp et al. (2008), and Lakdawalla et al. (2007) are the most significant for our work $\sqrt[6]{6}$ Berndt et al. (2003) examine drug prices, marketing, utilization, and over-the-counter to prescription switches occurring before and after generic entry for anti-ulcer drugs going off patent in the 1990s using wholesale price and sales data and retail scanner data. The authors find small increases in the branded manufacturers' prices after generic entry, decreases in branded marketing as generic entry approaches, and that the overall utilization of the therapeutic class falls - due in part to losing market share to over-the-counter products with the same active ingredients and brands. Huskamp et al. (2008) find that manufacturers release reformulations of anti-depressants (SSRIs) well before generic entry, reallocating promotion expenditures from the original molecule to the reformulation. This evidence suggests that manufacturers market reformulations as a means of extending patent life.

Most similar to our work are Caves et al. (1991) and Lakdawalla et al. (2007). Caves et al. (1991) examine 30 drugs losing patent expiration between 1976 and 1987 using first difference models and wholesale price and sales data. The authors find that branded prices increase in the period following patent expiration before generic entry, but then decrease as generic competitors enter the market. They also find decreases in advertising and overall utilization immediately before and after patent expiration. Lakdawalla et al. (2007) examine 101 molecules with expiring patents between 1992 and 2002 and find similar decreases in overall use and advertising prior to patent expiration. Their focus is to estimate the benefits from patent protection in terms of inefficient

\footnotetext{
${ }^{6}$ Other related work focuses on price responses to patent expiration. There is some disagreement about branded drug pricing following generic entry, but this research always finds large price decreases with time following generic entry and the number of generic competitors. Grabowski and Vernon (1992) investigate 18 drugs first facing generic entry between 1984 and 1988 and find that generic prices are initially lower than branded prices and continue to decrease. Unlike Caves et al. (1991), they find that branded prices increase at the same rate prior to and following generic entry. Frank and Salkever (1997) investigate 32 drugs going off patent during the 1980s and find that brand prices increase by 1 percent and generic prices decrease by 5.6 to 7.2 percent per generic competitor. Reiffen and Ward (2005) examine 31 drugs going off patent in the late 1980s and early 1990s and also find that increases in the number of generic competitors decreases generic prices. This literature reflects the substantial decrease in price and improvement in accessibility of drugs following patent expiration and generic entry.
} 
advertising. As such, they take a more structural approach estimating a 3SLS model of demand, supply, and advertising. Because their focus is not necessarily on the dynamics of utilization and prices in and around patent expiration, their empirical model allows for a limited number of discrete changes in these relationships focusing on one and five months after patent expiration. Our approach is more descriptive in the sense that we want to understand the dynamics of utilization and pricing of the molecule facing patent expiration, as well as other related molecules, both after and leading up to generic entry $!^{7}$

The market for pharmaceuticals and health insurance has changed considerably in the past 20 years. The sample in Caves et al. occurs almost entirely before the 1984 drug act, and generic drug use in the late 1980s was considerably lower than it is today. Pharmaceutical marketing is much more prevalent, as are cost-conscious health and drug insurance plans that discourage the use of branded drugs. As the brand market share for a given drug molecule after generic entry is very low, the choice between generic molecules or new patented products is increasingly significant for health care costs (Wallack et al., 2004). Our work makes three key contributions. First, we examine a set of recent patent expirations for blockbuster drugs, focusing on changes in utilization of branded reformulations. Second, we investigate whether utilization patterns vary with insurance type. Finally, we examine marketing as a mechanism for changes in utilization. We extend the analysis of Huskamp et al. (2008) both by considering molecules outside of anti-depressants and investigating the correspondence of changes in utilization and marketing.

The paper proceeds as follows. In Section 2, we discuss the FDA drug approval process, prescription benefit design, and pharmaceutical marketing. Section 3 discusses our data and the therapeutic classes on which we focus. Section 4 discusses our empirical approach. Section 5 discusses our results and Section [6 concludes.

\section{Institutional Background}

In this section, we describe the changes that have occurred in the regulation of generic drugs, prescription benefit designs, and advertising in the pharmaceutical industry.

\footnotetext{
${ }^{7}$ While Caves et al. (1991) and Berndt et al. 2003) do not find positive effects of price decreases following generic entry on the overall quantity of a molecule utilized, in other contexts researchers have found that decreased drug prices do lead to higher utilization. For example, Duggan and Scott-Morton (2010) find that the enactment of Medicare Part D in 2006 led to decreased prices for prescription drugs with high Medicare market shares, and that utilization increased as a result.
} 


\subsection{Patent Policy}

The treatment of generic drugs by the FDA and legislative bodies has varied considerably over the past 60 years. Prior to 1962, the approval process for generic drugs was shorter relative to that for new molecules (CBO, 1998). However, following the 1962 Kefauver Act, the FDA required that generic drugs be subjected to an approval process nearly as rigorous as that for new drug molecules, dissuading generic manufacturers from entering the market (Scherer, 2000). Additionally, following World War II many states enacted laws that prohibited the generic substitution of brand name drugs by pharmacists (Scherer, 2000). The net effect of these policies was that brand name drugs faced little generic competition following patent expiration, and the generic market share of those that did was significantly lower. The Congressional Budget Office reports that only 18 of 52 top selling drugs (that were non-antibiotics approved after 1962) faced generic competition in 1980, and that for drugs with generic equivalents, generics consisted only 12.7 percent of total prescriptions sold in retail pharmacies (CBO, 1998).

Barriers to generic entry were lowered significantly in the 1980s. State laws prohibiting generic substitution were all abolished by the mid-1980s (Grabowski and Vernon, 1986). Additionally, the Hatch-Waxman Act, passed in 1984, shortened the approval process for generic imitators of branded pioneer drugs whose patent was expiring. Under the Hatch-Waxman Act, generic imitators needed only to show that the generic version of a drug molecule was equivalent biologically to the pioneer molecule (Scherer, 2000). Branded manufacturers were also given longer exclusivity as part of the act, in order to account for the large portion of the patents' life taken up by the FDA approval process (Grabowski and Vernon, 1986). On net, the Hatch-Waxman Act substantially increased the utilization of generic drugs. The CBO cites 1997 IMS America data showing that the generic fraction of all tablets and capsules sold increased from 18.6 percent in 1984 to 42.6 percent in 1996 (CBO, 1998).

\subsection{Prescription Drug Benefit Designs}

While the generic share of overall prescriptions has increased considerably since Hatch-Waxman, total prescription drug expenditures have continued to rise. In nominal terms, total retail spending on prescription drugs increased from $\$ 40.3$ billion in 1990 to over $\$ 227.5$ billion in 2007 (Hartman et al. 2009b). Both private and public providers of health insurance have responded to increasing 
drug costs by implementing prescription drug benefit designs incentivizing use of cheaper generic drugs over more expensive brand name drugs.

Private insurers have incorporated tiered prescription benefit plans that require higher copayment for brand name drugs over generic drugs and mandatory substitution of generics. Such strategies have been shown to reduce prescription costs (Joyce et al., 2002, Malkin et al., 2004). While similar benefit designs have been implemented across private plans, there is evidence that the tiers are steeper in Health Maintenence Organizations (HMOs) than in traditional fee for service plans (Wallack et al., 2007). Additionally, HMO plans have smaller physician networks that exert more influence over physician prescribing behavior via formularies and educational programs (Wallack et al. 2004) and financial incentives and penalties (Scherer, 2000).

Public health insurance plans have also sought to reduce prescription costs. Some state Medicaid plans require prior authorization before filling certain prescriptions, mandate use of generic drugs, and in some cases require documentation that generic drugs are ineffective before covering brandname drugs; however, evidence is mixed on the extent to which such policies reduce brand name drug use and reduce costs (Cunningham, 2005, Fischer et al., 2007). Many Medicare Part D enrollees enroll in private plans that include multi-tiered copayment levels (Zhang et al. 2009).

The existing literature suggests that prescription benefit design may influence the use of generic versions of original molecules and reformulations after generic entry. In an extension of our main analysis, we estimate stratified regressions, dividing patients into Employer-HMO health insurance, Employer non-HMO health insurance, Medicare, Medicaid, and uninsured categories.

\subsection{Prescription Drug Advertising}

Concurrent to increased efforts to reduce pharmaceutical spending, pharmaceutical advertising has grown over the past two decades. Physician detailing and direct-to-consumer advertising (DTCA) are mainly performed by brand name companies and shift utilization away from generic drugs towards branded treatments. Expenditures are substantial. In 2007, pharmaceutical companies spent $\$ 10$ billion on promotional activity $(\mathrm{KFF}, 2008)$. Companies direct advertising to both physicians and consumers. Physician-directed advertising takes the form of either professional journal advertising ( $\$ 400$ million) or sales representative "detailing" ( $\$ 6.3$ billion). Changes to and the clarifying of direct-to-consumer advertising rules has lead to an increase in direct-to-consumer 
advertising, topping $\$ 3.7$ billion in 2007. Growth in advertising has also been substantial, especially in direct-to-consumer advertising. Palumbo and Mullins (2002) report that DTC advertising was a mere $\$ 12$ million in 1989 .

There is substantial evidence that advertising affects prescription drug utilization. Rosenthal et al. (2003) and Iizuka (2004) find that the main effect of direct-to-consumer advertising is to increase the overall market of a class of drug, rather than expand the market share of the individual drug that is advertised. Meyerhoefer and Zuvekas (2008) find similar market increases for anti-depressants, but that this happens through more patients using anti-depressants, rather than changes in anti-depressant utilization among users. Iizuka and Jin (2005) find that direct-toconsumer advertising increases doctor visits and that physician detailing influences what drug a doctor will prescribe. In summary, the existing literature suggests that pharmaceutical advertising, and physician detailing in particular, may have important effects on drug utilization patterns around pharmaceutical patent expiration and generic entry.

\section{Data}

Our analysis uses price and quantity measures derived from the Medical Expenditure Panel Survey, generic entry dates from the Food and Drug Administration (FDA), and promotion data from IMS Health. This section describes the construction of analytic data and the therapeutic classes included in the analysis.

\subsection{Generic entry dates}

Generic entry dates come from the FDA Center for Drug Evaluation and Research's Drugs@FDA electronic database, which provides approval information for all branded drugs and their therapeutic equivalents 8 These dates are provided, with the list of molecules in our sample, in Appendix Table 1.

\footnotetext{
${ }^{8}$ http://www.accessdata.fda.gov/scripts/cder/drugsatfda/
} 


\subsection{Prices, utilization, and controls}

We construct price and utilization measures and controls from the Medical Expenditure Panel Survey (MEPS). We describe this process in greater detail in Appendix A. Our analysis uses MEPS data from 1996 through 2007, comprising of 12 overlapping two-year interview panels (with only one year for the last panel). Each panel contains a nationally representative sample of approximately 16 thousand individuals who are interviewed over five interview rounds across the two years. We create biannual observations for each individual, and then take means within a product and six-month period (using MEPS person weights) such that the unit of analysis is product (or "molecule") by six-month period.

We construct six price measures. The first is the average total unit price for the branded version of a drug (by "total", we mean the amount paid to pharmacies across all payers). The second is the average total unit price across generic and branded versions of a drug (weighted by use). Finally, we construct a "lower bound" total unit price, defined as the average branded price prior to generic entry and the average generic price following. We also calculate "out-of-pocket" versions of each price measure. We convert the nominal unit prices to real prices using year 2000 dollars and then take the natural log. Average out-of-pocket prices are zero for some observations; thus, for all price outcomes, we add one before taking the natural log.

The main utilization measures indicate whether an individual was prescribed a drug molecule in a given six-month period, with separate variables for the branded version, generic version, or either version. In addition, we construct an indicator variable for whether an individual was prescribed a branded reformulation of each molecule. Utilization measures are then normalized by the fraction of the population using a molecule in the six months prior to generic entry in order to be comparable across molecules. 9

We create variables from the MEPS indicating whether individuals hold private HMO health plans, employer provided health insurance that is not from a HMO, Medicaid, Medicare, or are uninsured. Finally, we also compute demographic and health insurance characteristics of individuals obtaining each drug in each period. These controls include the fractions of users who are black non-Hispanic, Hispanic, aged 20 through 39, aged 40 through 59, and aged 60 or over.

\footnotetext{
${ }^{9}$ We do not take logs because of zeros.
} 


\subsection{Measures of promotion}

Advertising measures are derived from IMS Health Promotion Audits from January 1999 through December 2008. We construct: (1) product-specific quarterly costs of detailing, where detailing includes a discussion with a provider, a brief mention of the product, a drug fair, an educational presentation, a service visit, a telephone call, or other similar promotion, (2) product-specific costs for advertising in medical journals in each quarter, (3) the number of free samples in each quarter, and (4) total quarterly promotion costs (including all direct-to-consumer advertising, detailing, free samples, and other promotion).

\subsection{Therapeutic Classes}

Our sample includes prescription drugs in therapeutic classes treating depression, hypertension, high cholesterol, and type 2 diabetes. We chose prevalent chronic diseases that are (to varying degrees) controlled by long-term drug use. Our sample includes drugs in classes that are the most common first-line treatments for these diseases and are among the most prescribed of all drugs, with the highest expenditures. The specific drugs in our sample are displayed in Appendix Table 1.

Diabetes is a mounting public health concern. 25.8 million people- 8.3 percent of the U.S. population- had diabetes in 2010, with 1.9 million newly diagnosed adult (older than 19) cases (CDC, 2011). Poor control of diabetes can lead to or exacerbate other medical conditions including heart disease, stroke, and hypertension. In addition, diabetes is the leading cause of kidney failure, nontraumatic lower-limb amputations, and new cases of blindness among adults in the United States (CDC 2011). We investigate two first-line type 2 diabetes oral medication classes, used when diet and exercise are not sufficient to control blood glucose: biguanides and sulfonylureas. Sulfonylureas help stimulate insulin production in the pancreas, and thereby lower blood glucose, while Biguanides, a drug class primarily including metformin, increases sensitivity to blood glucose (Barnett and Braunstein, 2004). The first sulfonylurea received FDA approval in the mid-1980s and went off patent in the 1990s. Metformin received FDA approval in 1996 and went off patent in 2001. We will consider each class individually and drugs that are combinations of the two classes. These two classes of drugs, and metformin in particular, are among the most prescribed in the country. Over 4.5 million people in the US filled a metformin prescription in 2000, increasing to 
7.5 million people in 2006, putting metformin among the top ten drugs prescribed in each year 10

Hypertension, or high blood pressure, afflicts 31 percent of adults in the United States (CDC, 2010). Hypertension can cause or exacerbate coronary artery disease, stroke, heart failure, and renal disease (Awtry and Loscalzo, 2004). We focus on two common first- or second-line therapeutic classes for lowering blood pressure: ACE inhibitors and Angiotensin receptor blockers (ARB). These two classes are very similar in mechanism and effect (Fischer et al., 2007). However, ACE inhibitors lost patent protection starting in the mid-1990s, while ARBs are still under patent 11 ACE inhibitors are among the top drugs prescribed in the US. Lisinopril, a popular ACE inhibitor, was prescribed to over 11 million people in 200612

High blood cholesterol affects 16 percent of adults in the United States and is associated with a higher risk of heart disease (CDC, 2009). Statins form the primary pharmaceutical treatment for lowering LDL blood cholesterol and can help reduce deaths from heart disease ${ }^{13}$ The first statin, lovastatin, received FDA approval in 1987, and went off patent in 2001. However, numerous statins still have patent protection. Atorvastatin (Lipitor) was the third most prescribed drug in 2000, with over 7 million people in the US receiving prescriptions 14 By 2006, this number increased to almost 14 million people in the US in 2006, making Lipitor the most prescribed drug in the United States. Simvastatin and Vytorin were also in the top 25 drugs in terms of total expenditures throughout the last decade, with millions of Americans receiving prescriptions ${ }^{15}$

Depression affects 20 million people in the United States 16 We examine drugs in three common first-line therapeutic classes treating depression and anxiety: selective serotonin reuptake inhibitors (SSRIs), serotonin and norepinephrine reuptake inhibitors (SNRIs), and norepinephrine and dopamine reuptake inhibitors (NDRIs). Early antidepressants, fluoxetine (Prozac) and bupropion (Wellbutrin) obtained FDA approval in 1987 and 1985 respectively. Generics for these classes became available in the late 1990s and early 2000s. These molecules are also among the most prescribed drugs with the highest overall expenditures. Fluoxetine (Prozac) was prescribed to over 3 million people in 2000, with expenditures of almost 2 billion dollars. In 2006, escitalopram

\footnotetext{
${ }^{10}$ http://meps.ahrq.gov/

${ }^{11}$ As a result, while we mention ARBs, they are not included in the analysis.

${ }^{12}$ http://meps.ahrq.gov/

${ }^{13}$ http://www.cdc.gov/nchs/fastats/

${ }^{14}$ http://meps.ahrq.gov/

${ }^{15}$ http://meps.ahrq.gov/

${ }^{16}$ http://www.cdc.gov/nchs/fastats/
} 
(Lexapro) was prescribed to over 4.5 million people with total expenditures of almost 2 billion dollars, and sertraline (Zoloft) was prescribed to 4.3 million people with total expenditures of over 2 billion dollars 17

\section{Empirical Methodology}

Our empirical approach combines event study regressions and descriptive case studies. The event studies seek to identify how prices and utilization of both the "at-risk" molecule and patented reformulations change in response to, or expectation of, generic entry. Having established these empirical results, we next analyze how promotional activity corresponds with changes in prices and utilization around the time of generic entry using case studies.

The main sample for the event study analysis includes 35 drugs treating type 2 diabetes, hypertension, anxiety and depression, or high cholesterol that lose patent protection between 1996 and 2007 (listed in Appendix Table 1). Price outcomes are from the MEPS and include the average total price of the branded version of a drug, the average total price across branded and generic versions, a "lower-bound" total price that is the average branded price prior to generic entry and the average generic price after entry, as well as patient "out-of-pocket" versions of each measure. Utilization outcomes also come from the MEPS and include the probability of receiving the branded version of a drug, the probability of receiving the generic version, the probability of the branded or generic version, and the probability of a branded reformulation.

On the patient side, generic entry may represent a shift in accessibility as a drug molecule becomes less expensive with generic entry. For example, a drug molecule may move to a lower copayment tier or be added to a drug plan formulary. While this shift may not be completely unanticipated, we argue that this decrease in price can be considered exogenous from the patient's perspective. On the manufacturer's side, however, generic entry is hardly an unanticipated event. As discussed in the introduction, drug manufacturers respond to imminent generic entry by changing prices and marketing of branded drugs; thus, the impacts of generic entry come prior to the actual event. As such, simple pre-post comparisons are inadequate. Instead, we use event study models that flexibly trace changes in pricing and utilization over the life-cycle of the drug. This empirical strategy disentangles life-cycle effects from aggregate demand (or time) effects by exploiting

\footnotetext{
${ }^{17}$ http://meps.ahrq.gov/
} 
the differential timing of generic entry, as in (1):

$$
y_{m t}=\alpha+\beta_{t}+\mu_{m}+\gamma_{m} t+\sum_{k \neq-1, k \geqslant-10}^{k=5} \theta^{k} P O S T_{m t}^{k}+\eta X_{m t}+\epsilon_{m t},
$$

where $y_{m t}$ is the average price or utilization of molecule $m$ at time $t$ (where $\mathrm{t}$ represents a sixmonth period), $\alpha$ is a constant, $\beta_{t}$ are time fixed effects capturing general changes in prescription drug activity, $\mu_{m}$ are molecule fixed effects to control for time-invariant characteristics of molecule $m$ (e.g., variation in the baseline probabilities of being prescribed a particular molecule), $\gamma_{m t}$ are linear molecule time trends, and $X_{m t}$ are time-varying characteristics of patients being prescribed molecule $m$ at time $t$. The variables $P O S T^{-10}$ through $P O S T^{5}$ are indicator variables for 10 or more biannual periods prior to expansion through 5 or more biannual periods following entry, with the indicator $P O S T^{-1}$ omitted as the comparison period 18 . The associated $\theta$ coefficient estimates trace changes in outcomes prior to and following generic entry. In the reformulation analysis, $y_{m t}$ is defined as the fraction of the population using a reformulation, but POST is defined as the date when the original formulation faced generic competition. We calculate standard errors clustering on therapeutic class - six-month cells.

We specify the number of pre- and post-periods such that each parameter estimate is identified by the majority of the drugs facing generic competition. As many of the drugs in these therapeutic classes lost patent protection later in the sample period, there are more pre-periods than postperiods. We use the estimated fraction of the population using each molecule in the six months prior to generic entry as regression weights for each drug. Thus, our estimates show the average effect of generic entry on individuals using these classes of prescription drugs, rather than the average effect across drugs.

We estimate regressions stratifying by health insurance type to determine whether patterns of use vary with the structure of prescription drug coverage. We also estimate event studies stratifying by age. We test the robustness of our results by estimating separate event studies that (1) omit molecule specific time trends, (2) include therapeutic class by time fixed effects, (3) restrict the event study to a balanced panel, and (4) omit regression weights.

\footnotetext{
${ }^{18}$ We only examine five pre-generic entry biannual periods for the price analysis
} 


\section{Results}

\subsection{Summary statistics}

Table 1 displays summary statistics. The first column displays the mean characteristics of the person-biannual MEPS observations between 1996 and 2007, computed using MEPS sample weights. The second, third, and fourth columns show summary statistics for individuals using branded, generic, or any version of the drugs in our sample. These statistics show that 15.9 percent of the US population used the drugs in the sample over the sample period, illustrating the policy relevance of these therapeutic classes. Patients using the drugs in the sample are primarily covered by Medicare or private insurance. Most of the individuals with prescriptions in our data are older than 40; this is unsurprising, as the prevalences of type 2 diabetes, high cholesterol, and hypertension increase with age.

\subsection{Price Event Study Results}

Our first event studies display price trends in the years before and after generic entry. We estimate separate regressions for branded prices, the average price across branded and generic versions purchased, and a lower-bound price defined as the average branded price prior to generic entry, and the average generic price post-entry. We estimate equation (1), where the unit of observation is a molecule and six-month period. Prices are expressed in log-real terms. To account for zeros in out-of-pocket prices, we add one prior to taking natural logs. We regress price outcomes on pre- and post-generic entry indicator variables, biannual fixed effects, drug molecule fixed effects, characteristics of individuals using each molecule including race and ethnicity (fractions black non-Hispanic and Hispanic), health insurance status (fractions with Medicare, Medicaid, employerprovided HMO, and employer-provided non-HMO), age (fraction under 40, fraction 40 to 60, and fraction 60+), and molecule-specific time trends in the main specifications. Our sample includes the 35 molecules in the therapeutic classes of interest facing generic competition between 1996-2007. All estimates are weighted using the fraction of the population prescribed the molecule in the period prior to generic entry, and standard errors are clustered at the therapeutic class-biannual level.

Figures 1 and 2 graphically display regression estimates. In each figure, coefficient estimates are displayed as a solid line, and the dashed lines represent the 95 percent confidence interval for the 
coefficient estimates. The year prior to generic entry is omitted as the comparison period. Figures 1a and $\mathrm{b}$ show estimates for total prices of branded drugs, with and without molecule specific time trends. These estimates show little change in branded prices in the years prior to generic entry, and statistically insignificant increases after generic entry. Figures 1c and d show estimated changes in average prices before and after generic entry, of varying statistical significance. However, Figures 1e and $f$ trace out changes in "lower bound" prices around generic entry - defined as average branded prices prior to generic entry and average generic prices following generic entry- and show price decreases in the two years following generic entry that range from 10 to 15 percent. Figure 2 shows similar estimates for out-of-pocket prices. Figures $2 \mathrm{e}$ and f, most notably, show statistically significant decreases in generic out-of-pocket expenditures of 10 to 15 percent relative to pre-generic entry branded prices.

In sum, these estimates imply little change in branded prices leading up to generic entry and increased accessibility of drug molecules, with respect to price, after generic entry.

\subsection{Utilization Event Study Results}

Figure 3 displays pre- and post-generic entry trends in generic, branded, and overall drug molecule utilization. Utilization outcomes are constructed as the fraction of the US population using a molecule in each six-month period and are normalized by utilization in the six months prior to generic entry. In each case, the solid line indicates coefficient estimates and the dashed lines are 95 percent confidence intervals, with time since generic entry on the $\mathrm{x}$-axis and the period prior to generic entry omitted as the comparison period. The coefficient estimates can be interpreted as changes in utilization as a fraction of overall utilization in the six months before generic entry (after accounting for fixed effects, molecule-specific linear time trends, and other controls). Figure 3a shows that generic use increases to 40 percent of pre-entry branded use two years after entry. Figure $3 \mathrm{~b}$ shows that branded use begins decreasing in the two years prior to generic entry and decreases an additional 64 percent after two years of generic competition. In Figure 3c, total molecule use after generic entry - combining branded and generic drug use - decreases 20 percent in the two years after generic entry, and over 40 percent from two years prior to generic entry. This finding is surprising in light of evidence in Figures 1 and 2 that prices fall after generic entry. Assuming that branded and generic versions of a given molecule are equivalent, simple economic intuition predicts that total use would increase with the entry of cheaper generics, all else equal. 
Figure 4 exhibits reformulation event studies for 13 drugs in our sample that face generic entry and for which alternative formulations exist. These drugs, and their alternative formulations, are listed in Appendix Table 2. We define alternative formulations to include extended release versions of drugs and "combination" reformulations of original molecules with other drugs. The reformulation utilization measure is defined as the fraction of the population using a reformulation, divided by use of the original formulation in the six months prior to generic entry. The fractions of the population using the original formulation in the six months prior to generic entry are used as regression weights. Figure $4 \mathrm{a}$ exhibits pre- and post-generic entry trends in original branded formulation use for the "reformulation sample". As in the full sample case, the probability of obtaining the branded molecule begins falling in the two years before generic entry. Figure $4 \mathrm{~b}$ shows estimates from the reformulation event study, implying substantial growth in reformulation use in the years immediately prior to generic entry for the original formulation. Following entry, further increases are statistically insignificant, possibly because in some cases, the reformulations themselves lose patent protection. These estimates suggest that decreasing overall use of a molecule around generic entry may originate from patients shifting to branded reformulations.

\subsection{Utilization Event Study Extensions}

In Figures 5 and 6, we estimate separate event studies for patients with varying health insurance status. Figure 5 displays utilization event study results for patients with employer-provided HMO plans, employer provided non-HMO plans, Medicare, Medicaid, and no insurance. To the degree that HMOs and Medicaid may restrict access to newer branded drugs more than non-HMO employer provided plans, we expect smaller decreases in utilization for these groups prior to generic entry. However, Figures 5a-d show that patterns of total use are generally similar across all sources of insurance. Figure 6 displays estimates of reformulation use around generic entry stratified by health insurance type. Again, we predict that private non-HMO patients may exhibit greater use of branded reformulations than private HMO patients, Medicaid, or uninsured patients who have greater access to generics than newer branded drugs. However, the estimates are comparable across health insurance categories, with HMO patients exhibiting higher use of reformulations after generic entry.

The lack of differential effect across health insurance types suggests that these patterns of utilization exist in spite of benefit designs promoting use of generics to varying degrees. However, 
there are a couple caveats to this finding. First, we are not comparing patients across different benefit designs persay, but rather across reported type of health insurance. Second, health insurance status is not randomly assigned, so there may be selection processes by which patients selecting into HMOs, for example, have a higher propensity to shift to reformulations based on unobserved characteristics. As such, we interpret these findings as suggestive rather than conclusive.

In Figure 7, we examine whether patterns of drug use vary across younger and older patients. The estimates are less precise for younger patients, as older individuals are more likely to require medication to control hypertension, high cholesterol, and type 2 diabetes. Total use of original formulations falls at a similar rate prior to generic entry for younger (0-40 years old) and older (40-80 years old) patients.

Finally, we test the robustness of our main results to alternative specifications. Figure 8 displays results of alternative specifications for total use of original formulations. Figure 8a displays estimates not including molecule-specific trends, and shows more stable use prior to generic entry. More notable, however, is that the reduction in use is only marginally significant after up to one and a half years following entry and is then insignificant after this period. As will become evident with the case studies, controlling for molecule time trends is important as differential pre-generic entry trends appear to exist across molecules.

In Figure 8b, we include therapeutic-class-specific time effects. The comparison group in our main sample is defined as all other drug molecules not going off patent in the same period. This implicitly assumes that the utilization of other drugs is unaffected when one product faces generic competition. This assumption is implausible, especially within similar therapeutic classes treating the same condition. In fact, our results suggest that utilization of other branded drugs increases when a drug faces generic competition. By including class-time fixed effects, we control for classlevel changes in utilization, and thus the coefficient estimates are identified by changes in molecule use relative to other drugs in the sample from the same therapeutic class. The estimated effects of generic entry are larger, suggesting that utilization decreases relative to other drugs in the therapeutic class. Figure 8c restricts the sample to a "balanced panel", such that each molecule is present for 10 periods prior and 5 periods following generic entry, and finds similar results 19

In the main specifications, we use the fraction of the population using each drug molecule in the six months prior to generic entry as regression weights. In Figure 8d, we estimate the main

\footnotetext{
${ }^{19}$ The drugs in this balanced panel sample are listed in Appendix Table 1.
} 
specifications without weights, in order to detect whether a few heavily prescribed molecules are driving the results. Our estimates become less precise, but the magnitudes and overall pattern remains similar.

We are perform similar specification checks for the reformulation regressions in Figure 9. Omitting molecule specific trends (in Figure 9a) has little effect on estimated patterns of use; however, the standard errors become much larger. Allowing separate time fixed-effects for depression drugs relative to other classes (combining hypertension, cholesterol, and diabetes drugs) in Figure 9b does not substantively change our estimates, but leads to larger standard errors. Finally, we use the fraction of the population using the original formulation in the six months prior to generic entry as regression weights in our main specification. These weights vary considerably, from over 1 percent for metformin to only one-tenth of 1 percent for other molecules. Figure 9c shows that our estimates are extremely sensitive to including these weights, and that the main estimates are driven primarily by the more heavily used molecules.

\subsection{Case studies}

The event studies show that despite the decrease in price after generic entry, total use of an original formulation falls. We also find that use of branded reformulations increases, suggesting that patients shift from original formulations to newer branded reformulations. In this section, we examine specific case studies, and provide suggestive evidence on the role played by marketing.

The first case study is for metformin, a blockbuster medication for type 2 diabetes. Figure 10a shows prices of metformin increasing in real terms throughout the late 1990s and in 2000, and then leveling off in the two years prior to generic entry, after which generic prices fall in real terms. ${ }^{20}$ However, Figure 10b shows use of branded meformin increasing until 2000, and then falling in the two years prior to generic entry, seemingly unrelated to price. In Figure 10c, the entry and increased use of branded reformulations of metformin coincides with the decreased use of the branded original formulation prior to generic entry. Figures 10d and e show that these effects are likely driven by marketing; detailing costs and free samples for brand name metformin (Glucophage) and brand name metformin extended-release (Glucophage XR) mirror changes in

\footnotetext{
${ }^{20}$ There is a large drop in price in 2007 . We are able to match our total expenditure estimates with those from AHRQ for few molecules, so we believe this to be real. We hypothesize this is due to increased availability of generics through retail stores such as Wal-Mart, which began selling discounted generic drugs in 2006
} 
utilization of these products. Finally, medical journal advertising for Glucophage XR spikes prior to generic entry for extended release metformin in 2003.

Figure 11 shows prices and use of simvastatin (brand name Zocor), a popular medication used for lowering high cholesterol. Simvastatin received FDA approval in 1991 and faced generic competition starting in June 2006. Figure 11a shows little change in branded prices prior to generic entry and large decreases in the price of generic simvastatin after generic entry. Figure 11b shows decreased use of branded simvastatin in the two years prior to generic entry, and Figure 11c shows that this corresponded with increased use of Vytorin, a patented combination of simvastatin with ezetimibe (another cholesterol drug) also manufactured by Merck. Figures 11d and e exhibit large increases in detailing and samples of Vytorin corresponding with decreased simvastatin and increased simvastatin+ezetimbe use. In addition, Figure 11f shows a spike in medical journal advertising for Vytorin corresponding with changes in Zocor and Vytorin use.

Figure 12 displays prices and use of bupropion sustained release (SR). Bupropion SR (marketed as Wellbutrin SR) received FDA approval in 1999 and was the second of three Wellbutrin products released on the market. Prior to Wellbutrin SR facing generic competition in 2003, Wellbutrin XL (taken once daily) was released with exclusivity through 2008 21 Figure 12a shows average total "lower bound" unit prices for bupropion SR over the sample period. Branded prices for bupropion SR increased prior to generic entry. Generic prices oscillate after generic entry but were generally equivalent or lower in real terms (except for 2005). Despite relatively stable prices, total use of bupropion SR falls substantially after generic entry (in Figure 12c). Figure 12d shows that the decreased use of branded bupropion SR corresponded with the entry and increased use of branded bupropion XL (Wellbutrin XL). Furthermore, in Figure 12d and e, this occurred simultaneously with decreased advertising and samples for branded bupropion SR and increased advertising samples for bupropion XL. In addition, Figure $12 \mathrm{f}$ shows a sharp and transitory increase in medical journal advertising for Wellbutrin XL, immediately following generic entry for Wellbutrin SR.

Finally, Figure 13 displays prices and use of another popular SSRI, citalopram. Citalopram received FDA approval in 1998 and was marketed by Forest Labs under the brand name Celexa. Figures 13a show stable prices (in 2000 dollars) for citalopram in the years prior to generic entry and following generic entry (until 2007). Figure 13b shows citalopram use steadily increasing from release in 1999 until 2002 when nearly one percent of the population obtained Celexa. Between

\footnotetext{
${ }^{21}$ The original bupropion was administered three times daily, Bupropion SR two times daily, and bupropion XL one time daily.
} 
2002 and 2004, however, citalopram use fell almost 50 percent. Simultaneously, the FDA approved another SSRI marked by Forest Labs, escitalopram (brand name Lexapro). Escitalopram is the enantiomer, or molecular mirror image, of citalopram. Figure 13c shows the utilization of branded citalopram and branded escitalopram. The rapid take-up of escitalopram coincides almost exactly with the decrease in the use of citalopram, suggesting that patients and physicians were persuaded to shift from citalopram to escitalopram. Figures 13d-f support this hypothesis; patterns in detailing, samples, and total promotion (including physician detailing, professional promotion, and direct-toconsumer advertising) for Celexa and Lexapro coincide with changes in use.

These case studies confirm that use of medications falls in the years immediately before and after generic entry, and that this happens even when prices fall or are stable in real terms. However, we also find that this decreased use coincides with branded drug manufacturers unveiling new formulations of molecules facing generic entry. Examining advertising data, it appears that manufactures also shift marketing efforts to the new formulations. This behavior effectively extends the exclusivity of the original molecule and appears to reduce use of generics.

\section{Conclusions}

We use case studies, event study models, and data from the Medical Expenditure Panel Survey to estimate the effects of generic entry on prices and utilization of blockbuster drugs treating hypertension, high cholesterol, depression, and type 2 diabetes. We examine how generic entry effects differ across individuals with different sources of health insurance. We find that generic prices fall relative to branded prices, and that branded company market share falls rapidly after the entry of generic competition. However, we also see that the utilization of the branded molecule begins to fall prior to generic entry, and that this coincides with increased utilization of branded reformulations. We find suggestive evidence that these patterns exist across individuals with different sources of health insurance. Finally, case study evidence implies that marketing to physicians and patients drives these patterns of use.

The purpose of pharmaceutical patent policy is to optimally incentivize and reward drug manufacturers for innovation, while protecting society from excessive deadweight loss coming from monopoly pricing. We show evidence that rather than society enjoying the consumer surplus from competitive pricing after generic entry, utilization is shifted towards other still-patented drugs. 
We remain agnostic on the extra benefit from the reformulations and newer drugs (even patented reformulations that are combinations of two drugs that are both available generically could improve patient compliance). Comparative effectiveness analysis is necessary on a case-by-case basis to make such determinations. However, to the degree that some new and patented drugs offer little improvement in health outcomes relative to the generic drugs (and at considerable expense), current pharmaceutical patent policy may both discourage true innovation and prolong monopoly rents by granting exclusivity to products very similar to those losing patent exclusivity. As policymakers currently seek to control health care costs, expanding polices promoting use of generic pharmaceuticals may be fertile ground for achieving savings. 


\section{References}

Adler, J. L., W. Jannetti, D. Schneider, J. Zhang, R. Palmer, and G. Notario (2000): "Phase III, randomized, double-blind study of clarithromycin extended-release and immediaterelease formulations in the treatment of patients with acute exacerbation of chronic bronchitis," Clinical Therapeutics, 22, 1410 - 1420.

Awtry, E. H. And J. Loscalzo (2004): "Vascular Diseases and Hypertension," in Cecil Essentials of Medicine, ed. by C. C. J. Carpenter, R. C. Griggs, and J. Loscalzo, W.B. Saunders, 6th ed.

Barnett, P. S. And G. D. Braunstein (2004): "Diabetes Mellitus," in Cecil Essentials of Medicine, ed. by C. C. J. Carpenter, R. C. Griggs, and J. Loscalzo, W.B. Saunders, 6th ed.

Berndt, E. R., M. K. Kyle, And D. Ling (2003): "The long shadow of patent expiration: generic entry and RX-OTC switches," in Scanner data and price indexes, University of Chicago Press.

Caves, R. E., M. D. Whinston, M. A. Hurwitz, A. Pakes, and P. Temin (1991): "Patent expiration, entry, and competition in the U.S. pharmaceutical industry," Brookings Papers on Economic Activity, 1-66.

CBO (1998): "How increased competition from generic drugs has affected prices and returns in the pharmaceutical industry." Tech. rep., Congressional Budget Office.

CDC (2009): "Cholesterol Fact Sheet," Tech. rep., Center for Disease Control and Prevention.

— (2010): "High Blood Pressure Fact Sheet," Tech. rep., Center for Disease Control and Prevention.

(2011): "National diabetes fact sheet: national estimates and general information on diabetes and prediabetes in the United States," Tech. rep., Centers for Disease Control and Prevention.

Cunningham, P. J. (2005): "Medicaid Cost Containment and Access to Prescription Drugs," Health Affairs, 24, 780-789.

Dopfner, M., W. D. Gerber, T. Banaschewski, D. Breuer, F. J. Freisleder, G. Gerber-von Muller, M. Gunter, F. Hassler, C. Ose, A. Rothenberger, 
K. Schmeck, J. Sinzig, C. Stadler, H. Uebel, and G. Lehmkuhl (2004): "Comparative efficacy of once-a-day extended-release methylphenidate, two-times-daily immediate-release methylphenidate, and placebo in a laboratory school setting," European Child ${ }^{6}$ Adolescent Psychiatry, 13, i93-i101, 10.1007/s00787-004-1009-3.

Duggan, M. And F. Scott-Morton (2010): "The Impact of Medicare Part D on Pharmaceutical Prices and Utilization," American Economic Review, 100, 590-607.

Fischer, M. A., N. K. Choudry, And W. C. Winkelmayer (2007): "Impact of Medicaid Prior Authorization on Angiotensin-Receptor Blckers: Can Policy Promote Rational Prescribing?" Health Affairs, 26, 800-807.

Frank, R. G. And D. S. Salkever (1997): “Generic Entry and the Pricing of Pharmaceuticals," Journal of Economics and Management Strategy, 6, 75-90.

Grabowski, H. G. And J. M. Vernon (1986): "Longer Patents for Lower Imitation Barriers: The 1984 Drug Act," American Economic Review Papers and Proceedings, 76, 195-198.

(1992): "Brand loyalty, entry, and price competition in pharmaceuticals after the 1984 Drug Act," Journal of Law and Economics, 35, 331-350.

Hartman, M., A. Martin, P. McDonnel, And A. Catlin (2009a): "National health spending in 2007: slower drug spending contributes to lowest rate of overall growth since 1998," Health Affairs, 28, 246-261.

Hartman, M., A. Martin, P. McDonnell, And A. Catlin (2009b): "National Health Spending In 2007: Slower Drug Spending Contributes To Lowest Rate Of Overall Growth Since 1990," HealthAffairs, 28, 246-261.

Huskamp, H. A., J. M. Donohue, C. Koss, E. R. Berndt, and R. G. Frank (2008): "Generic Entry, Reformulations, and Promotion of SSRIs," Pharmacoeconomics, 7, 603-616.

IIzukA, T. (2004): "What Explains the Use of Direct-to-Consumer Advertising of Prescription Drugs?" Journal of Industrial Economics, 52, 349-379.

IIZUKA, T. AND G. Z. Jin (2005): "The Effect of Prescription Drug Advertising on Doctor Visits," Journals of Economics and Management Strategy, 14, 701-727. 
Joyce, G. F., J. J. Escarce, M. D. Solomon, and D. P. Goldman (2002): "Employer Drug Benefit Plans and Spending on Prescription Drugs," Journal of the American Medical Association, 288, 1733-1739.

KFF (2008): "Prescription Drug Trends," Tech. rep., Kaiser Family Foundation.

Lakdawalla, D., T. Philipson, And W. Y. Richard (2007): "Intellectual Property and Marketing," Tech. rep., National Bureau of Economic Research, Cambridge, MA.

Lundy, J. (2010): "Prescription Drug Trends," Tech. rep., Kaiser Family Foundation.

Malkin, J. D., D. P. Goldman, and G. F. Joyce (2004): "The Changing Face Of Pharmacy Benefit Design," Health Affairs, 23, 194-199.

Meyerhoefer, C. D. And S. H. Zuvekas (2008): "The Shape of Demand: What Does It Tell Us about Direct-to-Consumer Marketing of Antidepressants?" The B.E. Journal of Economic Analysis 85 Policy, Advances, 8, 701-727.

Palumbo, F. B. And C. D. Mullins (2002): "The Development of Direct-to-Consumer Prescription Drug Advertising Regulation," Food and Drug Law Journal, 57, 423-443.

PeruccA, E. (2009): “Trends in Retail Prescription Expenditures," Epilepsy Curr., 9, 153-157.

Reiffen, D. And M. R. WARd (2005): "Generic Drug Industry Dynamics," The Review of Economics and Statistics, 87, 37-49.

Rosenthal, M. B., E. R. Berndt, J. M. Donohue, A. M. Epstein, and R. G. Frank (2003): "Demand Effects of Recent Changes in Prescription Drug Promotion," Forum for Health Economics and Policy, 6, 1-26.

Rudolph, R. L. AND A. D. FeIger (1999): "A double-blind, randomized, placebo-controlled trial of once-daily venlafaxine extended release (XR) and fluoxetine for the treatment of depression," Journal of Affective Disorders, 56, 171 - 181.

Scherer, F. M. (2000): "The Pharmaceutical Industry," in Handbook of Health Economics, ed. by A. J. Culyer and J. P. Newhouse, Elsevier.

Schondelmeyer, S. W. And J. Thomas (1990): “Trends in Retail Prescription Expenditures," Health Affairs, 9, 131-145. 
Schwartz, S., V. Fonseca, B. Berner, M. Cramer, Y.-K. Chiang, and A. Lewin (April 2006): "Efficacy, Tolerability, and Safety of a Novel Once-Daily Extended-Release Metformin in Patients With Type 2 Diabetes," Diabetes Care, 29, 759-764.

Swanson, J. M., S. B. Wigal, T. Wigal, E. Sonuga-Barke, L. L. Greenhill, J. Biederman, S. Kollins, A. S. Nguyen, H. H. DeCory, S. J. Hirshe Dirksen, S. J. Hatch, AND the COMACS Study Group (2004): "A Comparison of Once-Daily Extended-Release Methylphenidate Formulations in Children With Attention-Deficit/Hyperactivity Disorder in the Laboratory School (The Comacs Study)," Pediatrics, 113, e206-216.

Wallack, S. S., C. P. Thomas, T. C. Martin, and A. Ryan (2007): "Differences in Prescription Drug Use in HMO and Self-insured Health Plans," Medical Care Research and Review, 64, $98-116$.

Wallack, S. S., D. B. Weinberg, and C. P. Thomas (2004): "Health Plans' Strategies to Control Prescription Drug Spending," Health Affairs, 23, 141-148.

Zhang, Y., J. M. Donohue, J. P. Newhouse, and J. R. Lave (2009): "The Effects Of The Coverage Gap On Drug Spending: A Closer Look At Medicare Part D," Health Affairs, 28, $317-325$. 


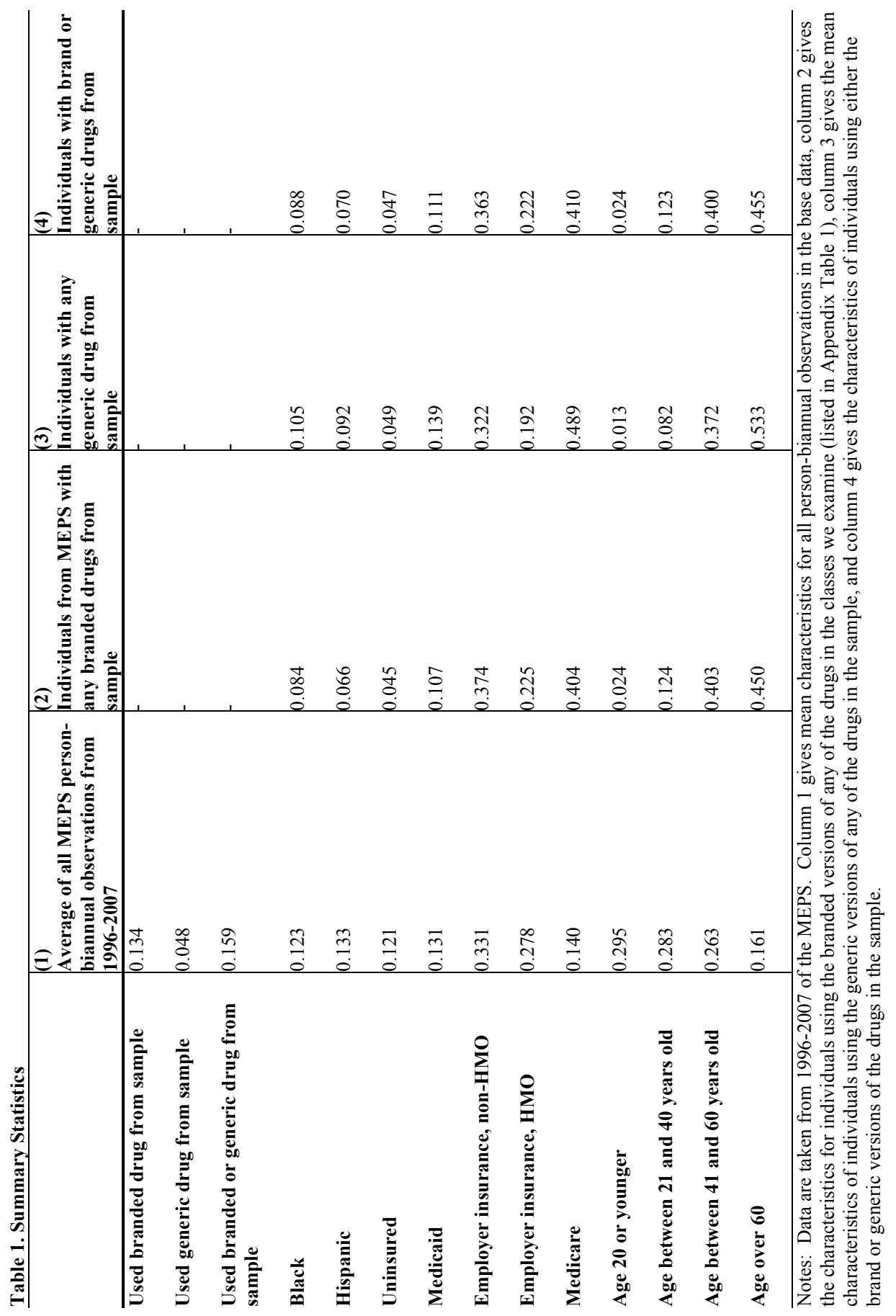


Figure 1: Total price event study results

(a) Brand prices

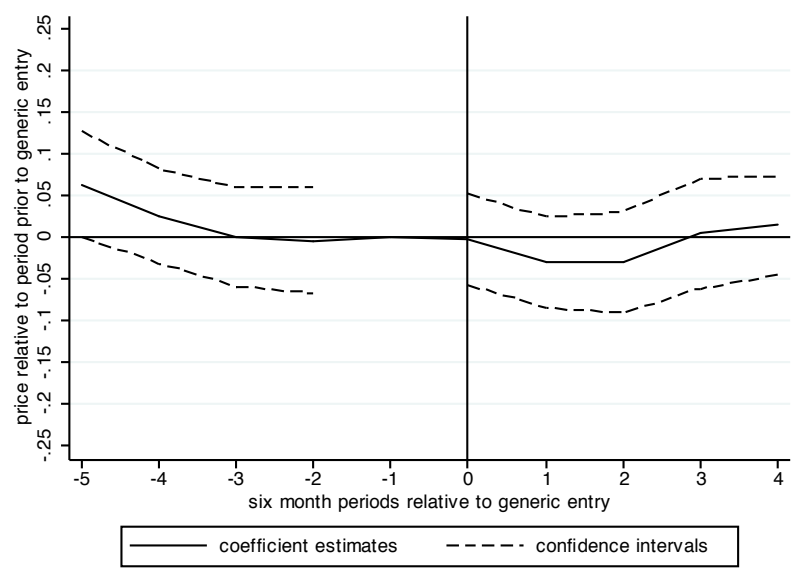

(c) Average prices

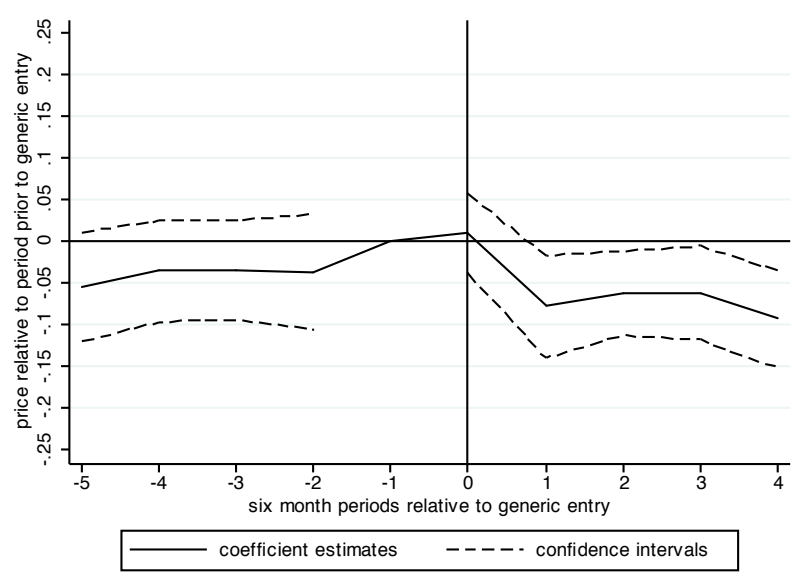

(e) Lower bound prices

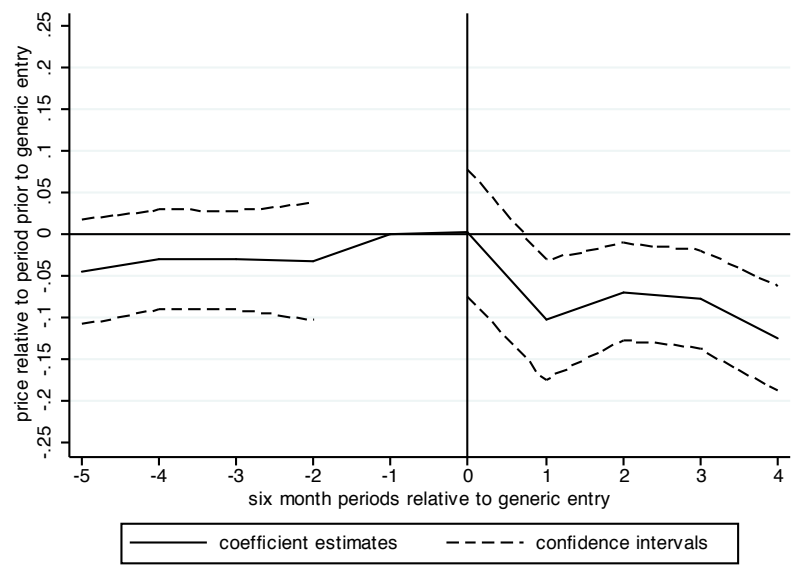

(b) Brand prices, w/molecule-specific trends

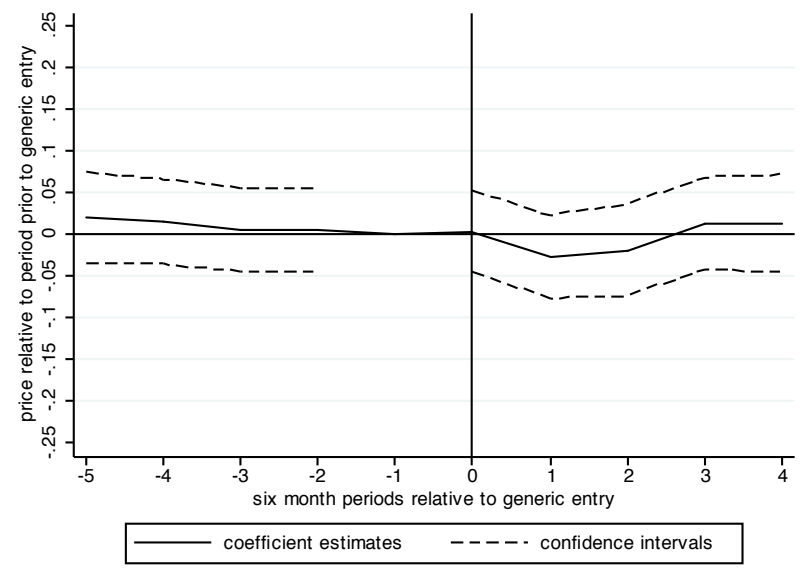

(d) Average prices, w/ molecule-specific trends

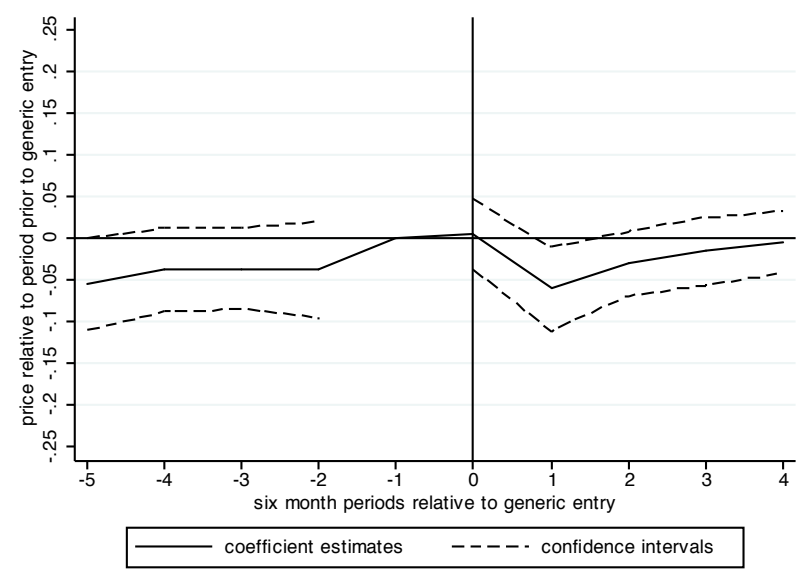

(f) Lower bound prices, w/ molecule-specific trends

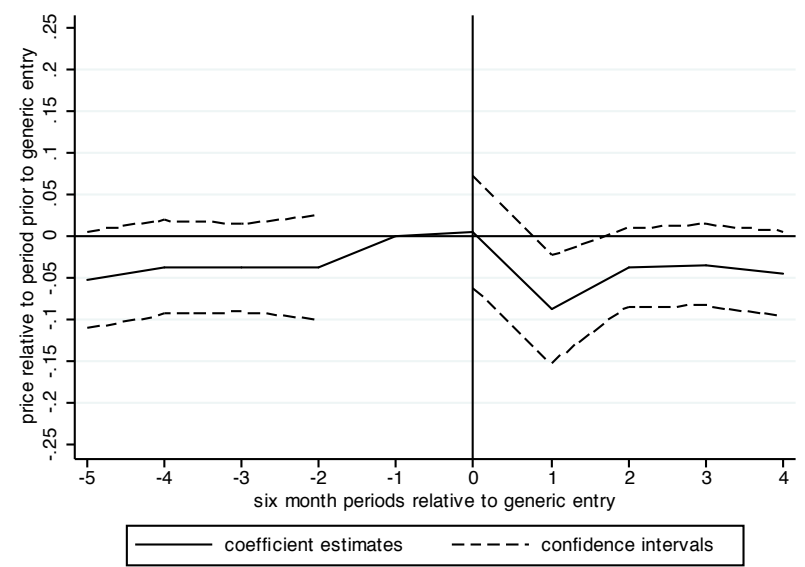

Notes: Solid lines are coefficient estimates, dashed lines are 95 percent confidence intervals. Standard errors clustered at the therapeutic class and six month period. Vertical line indicates generic entry. 
Figure 2: Out-of-pocket event study results

(a) Brand prices

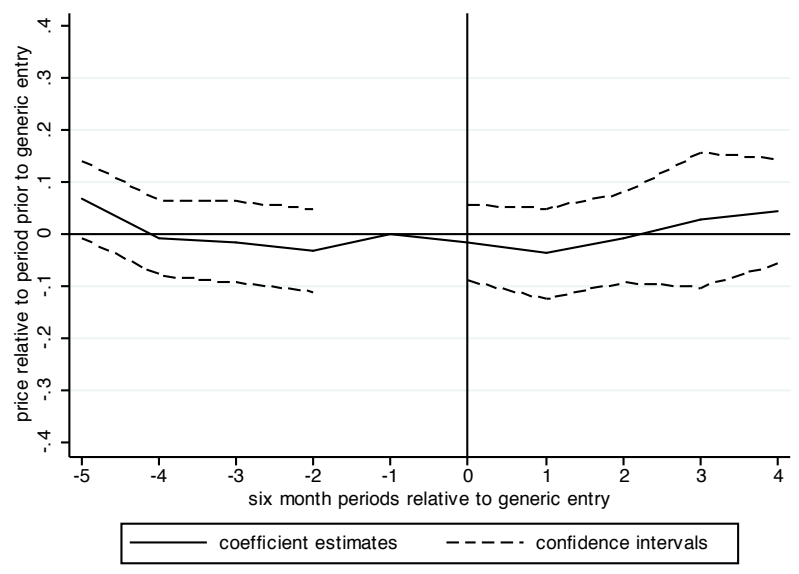

(c) Average prices

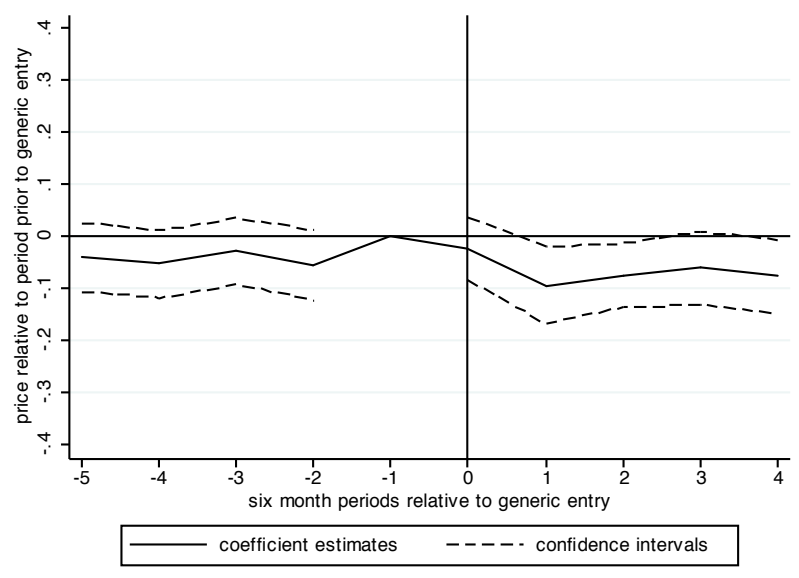

(e) Lower bound prices

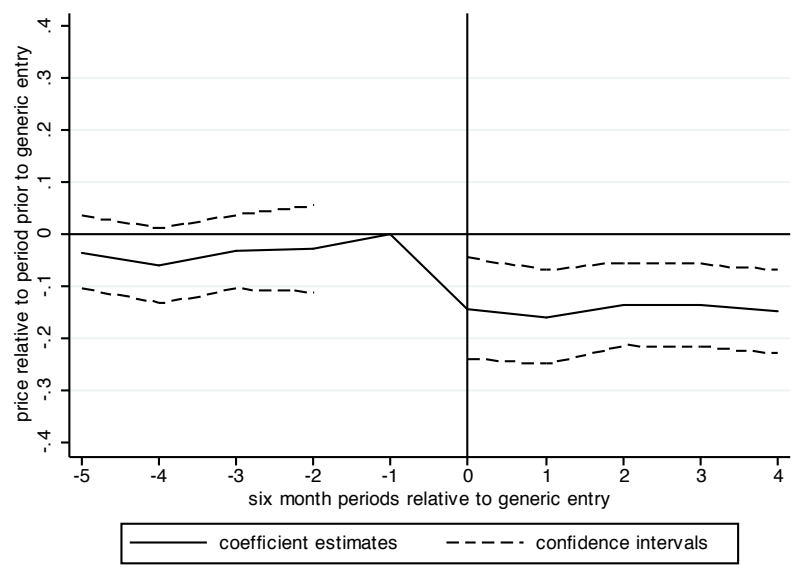

(b) Brand prices, w/molecule-specific trends

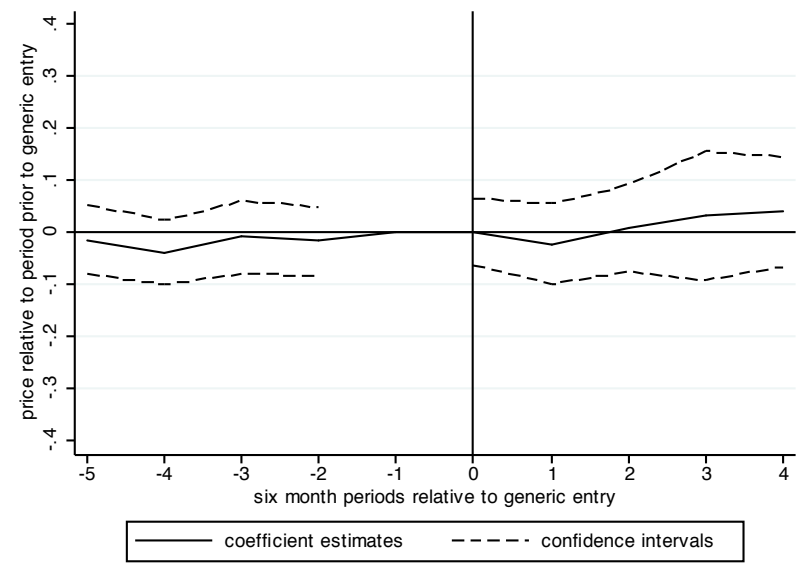

(d) Average prices, w/ molecule-specific trends

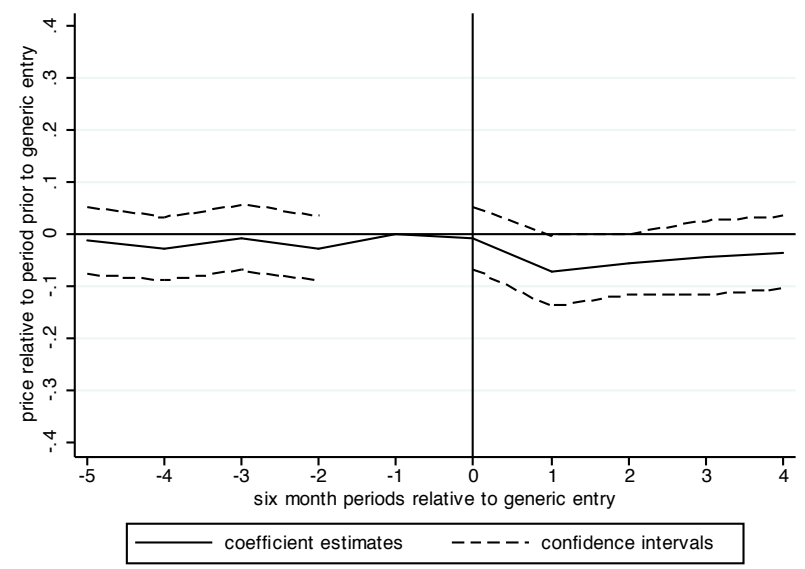

(f) Lower bound prices, w/ molecule-specific trends

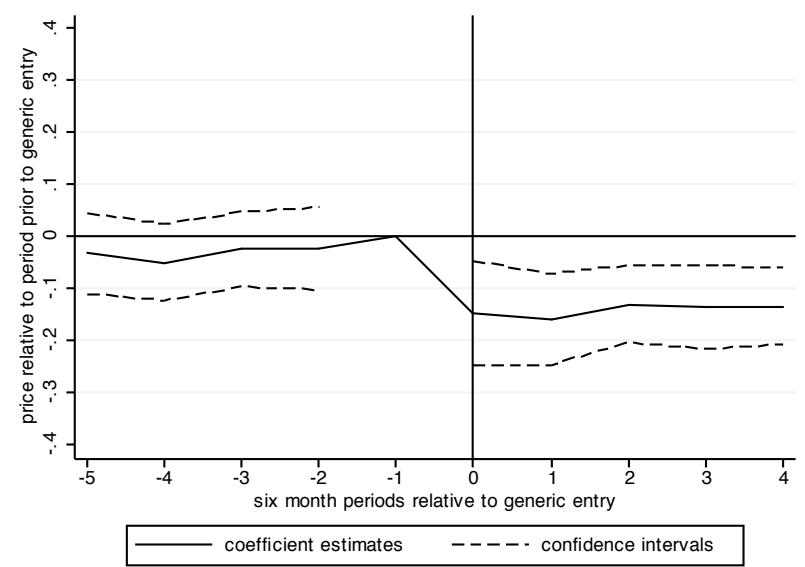

Notes: Solid lines are coefficient estimates, dashed lines are 95 percent confidence intervals. Standard errors clustered at the therapeutic class and six month period. Vertical line indicates generic entry. 
Figure 3: Generic entry utilization event studies

(a) Generic molecule utilization

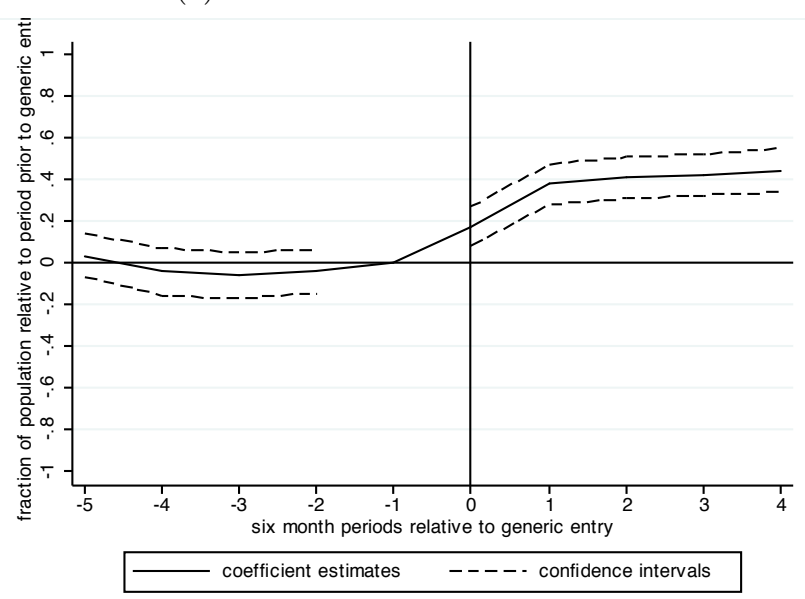

(c) Total molecule utilization

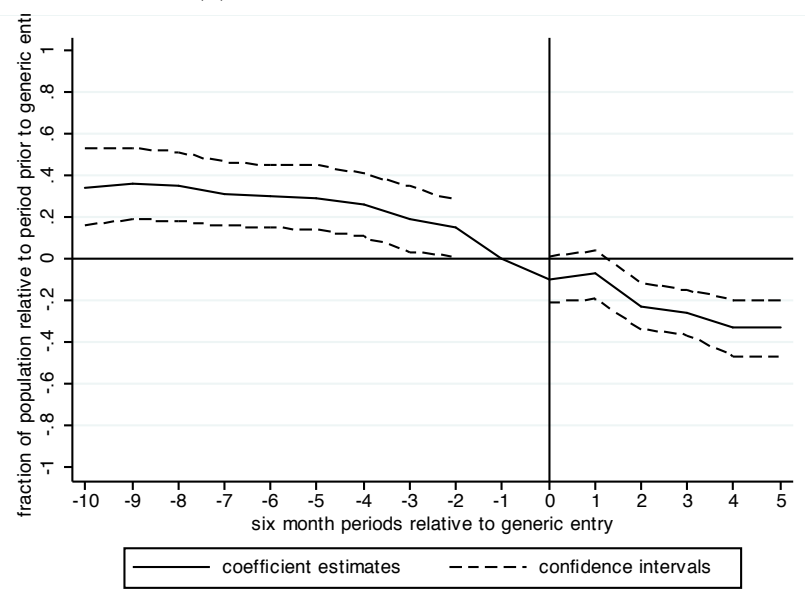

(b) Branded molecule utilization

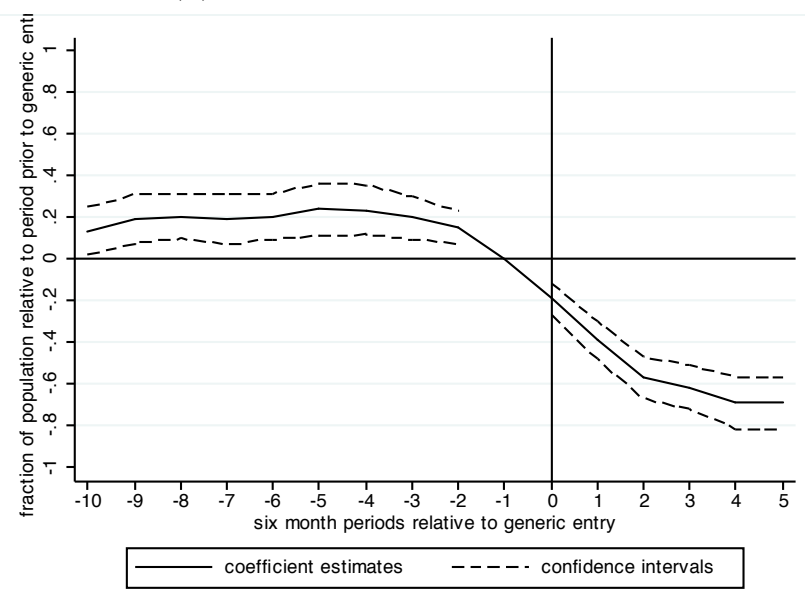

Notes: Solid lines are coefficient estimates, dashed lines are 95 percent confidence intervals. Standard errors clustered at the therapeutic class and six month period. Vertical line indicates generic entry. 
Figure 4: Branded alternative formulations of drug molecules facing generic entrants

(a) Utilization of original branded molecule

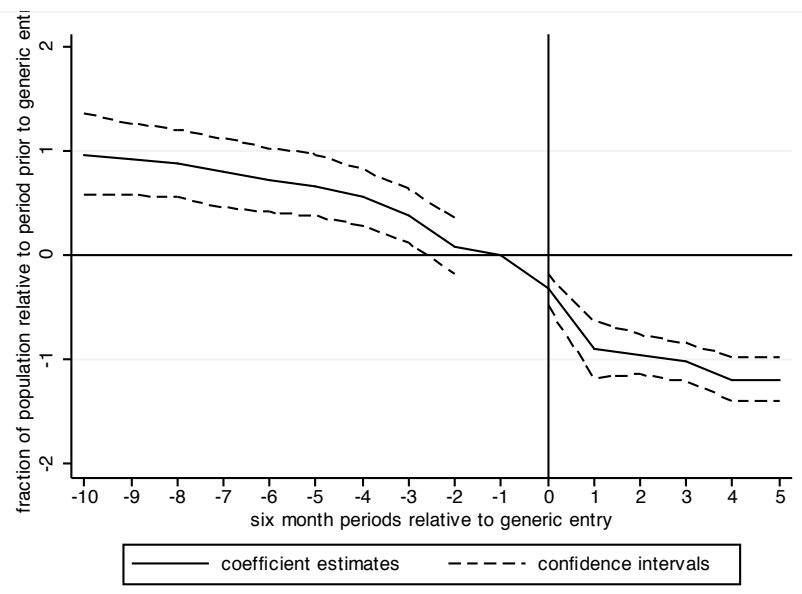

(b) Use of branded alternative formulations

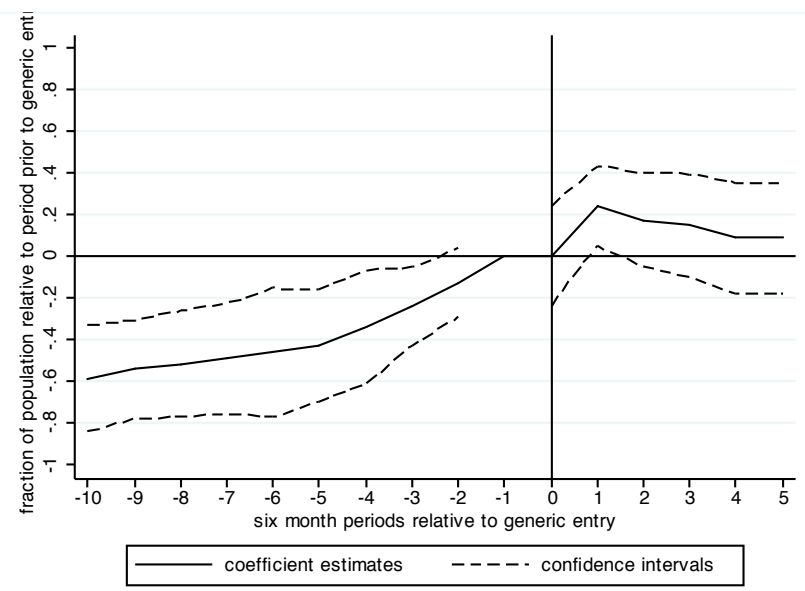

Notes: Solid lines are coefficient estimates, dashed lines are 95 percent confidence intervals. Standard errors clustered at the therapeutic class and six month period. Vertical line indicates generic entry. 
Figure 5: Total molecule use by health insurance type

(a) Employer-provided insurance, HMO

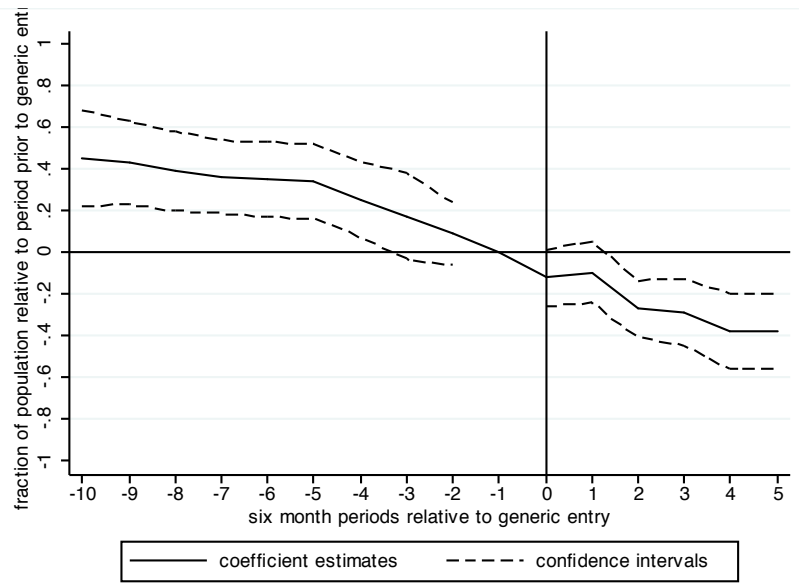

(c) Medicare

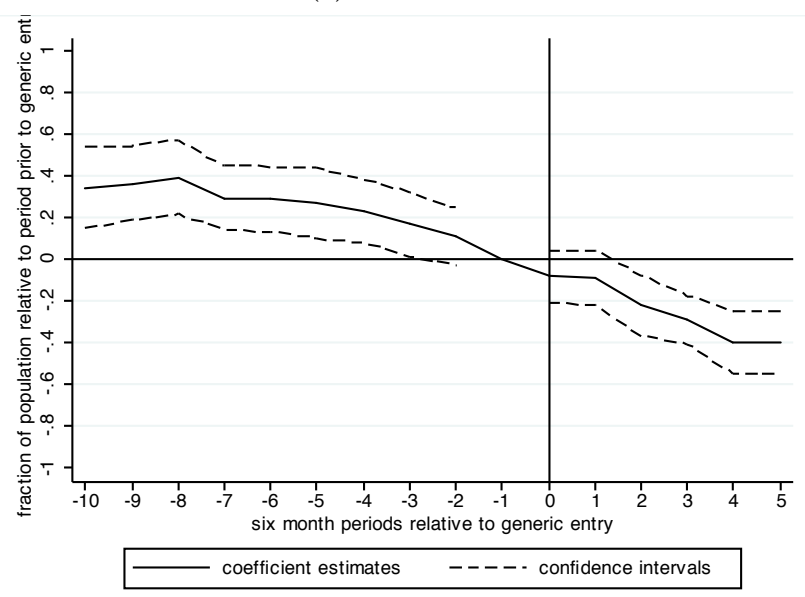

(e) Uninsured

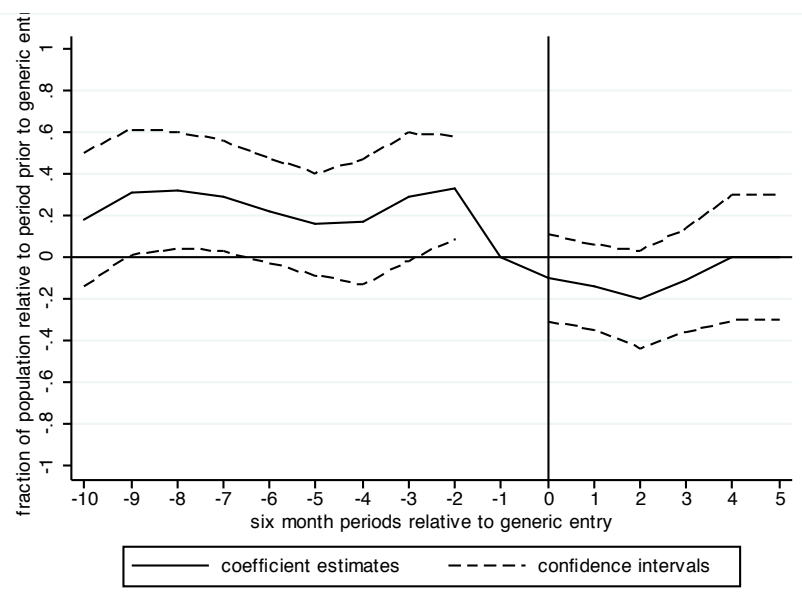

(b) Employer provided insurance, non-HMO

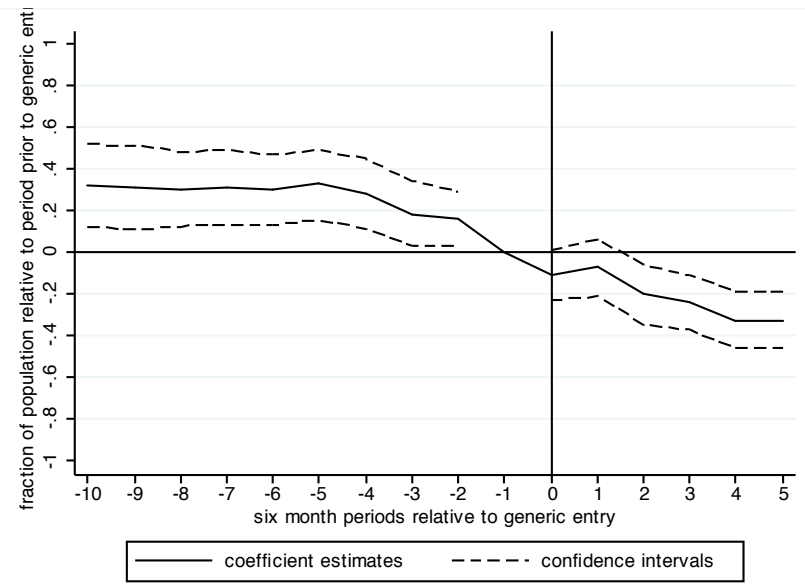

(d) Medicaid

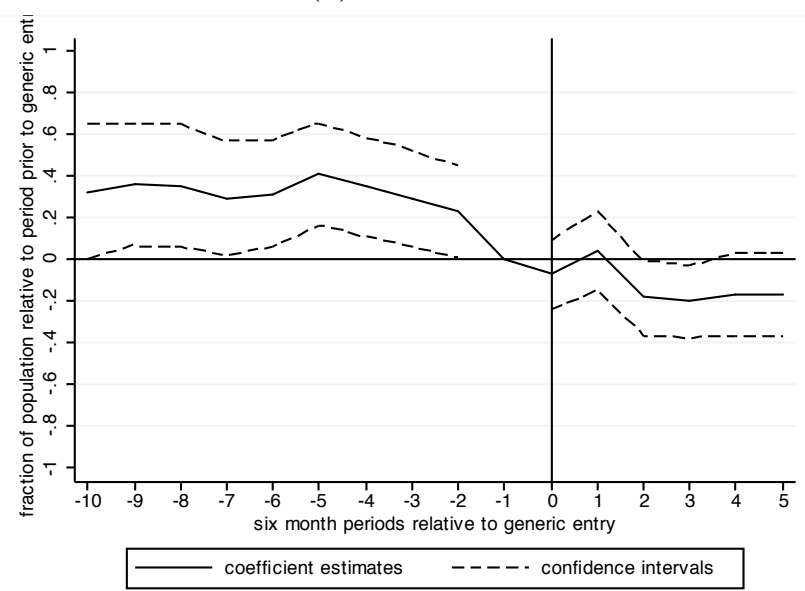

Notes: Solid lines are coefficient estimates, dashed lines are 95 percent confidence intervals. Standard errors clustered at the therapeutic class and six month period. Vertical line indicates generic entry. 
Figure 6: Use of reformulations by health insurance type

(a) Employer-provided insurance, HMO

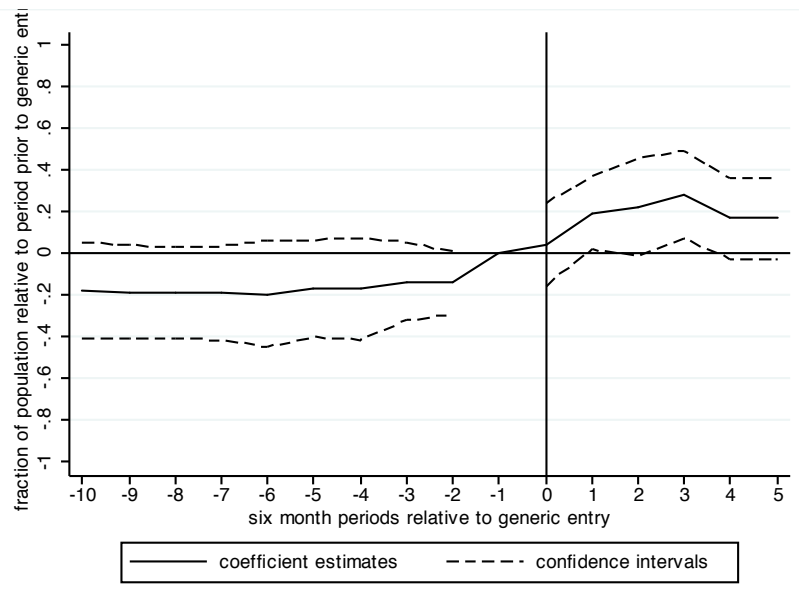

(c) Medicaid

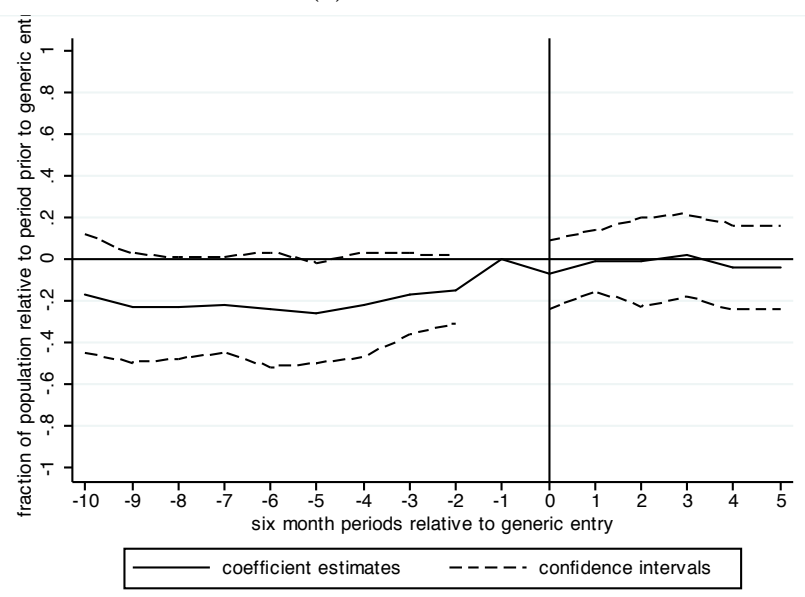

(e) Uninsured

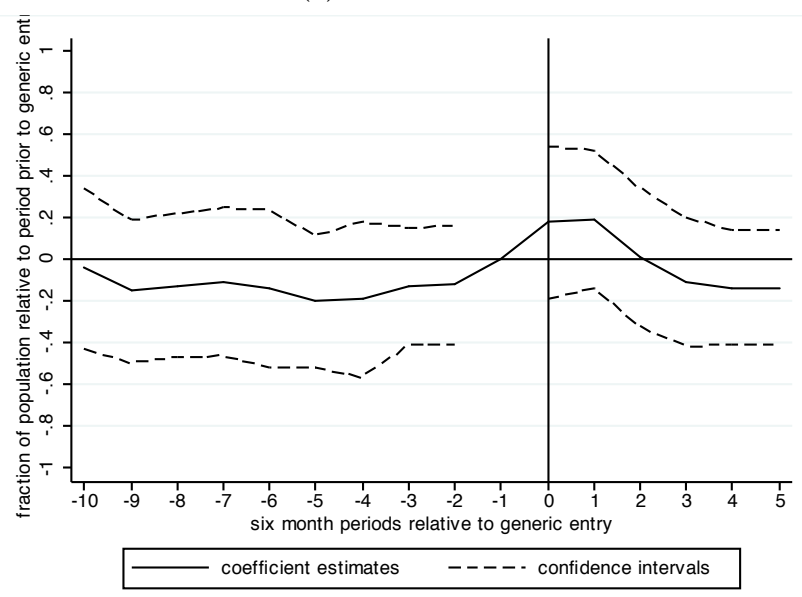

(b) Employer provided insurance, non-HMO

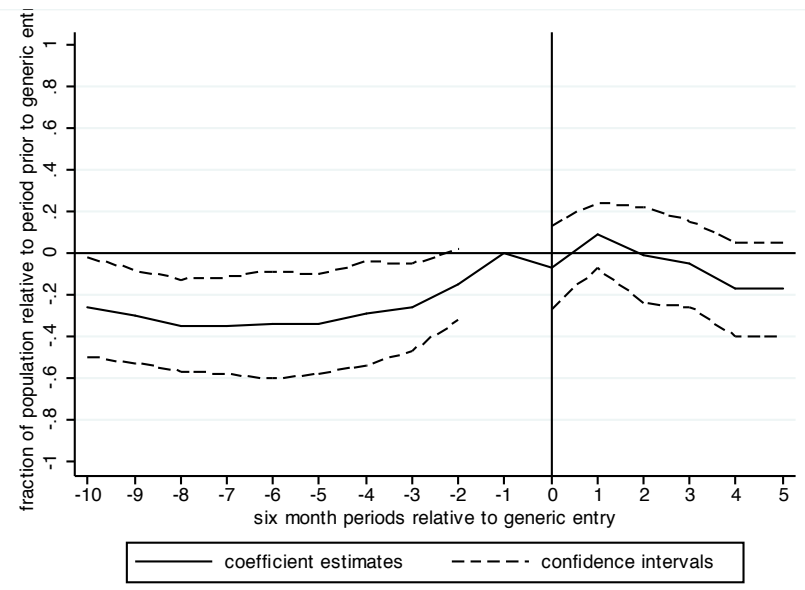

(d) Medicare

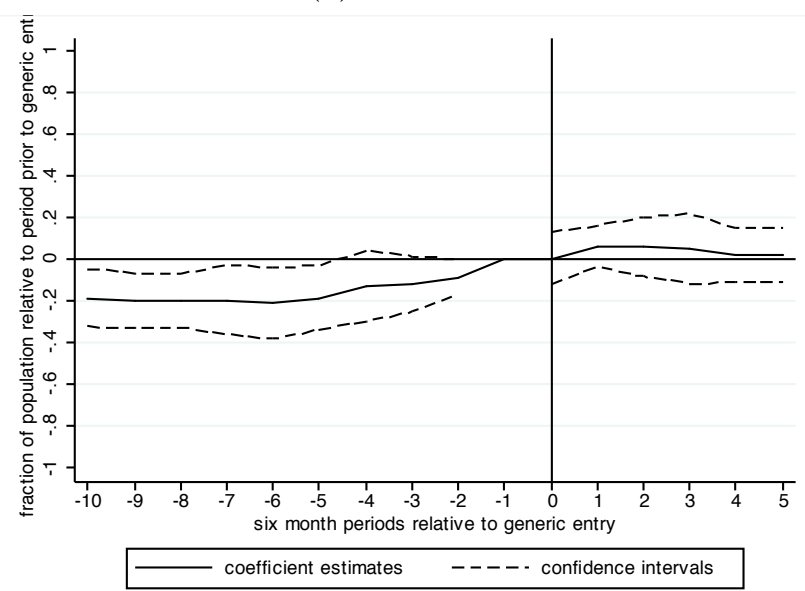

Notes: Solid lines are coefficient estimates, dashed lines are 95 percent confidence intervals. Standard errors clustered at the therapeutic class and six month period. Vertical line indicates generic entry. 
Figure 7: Drug use by age group

(a) Total use of original molecule, ages 0-40

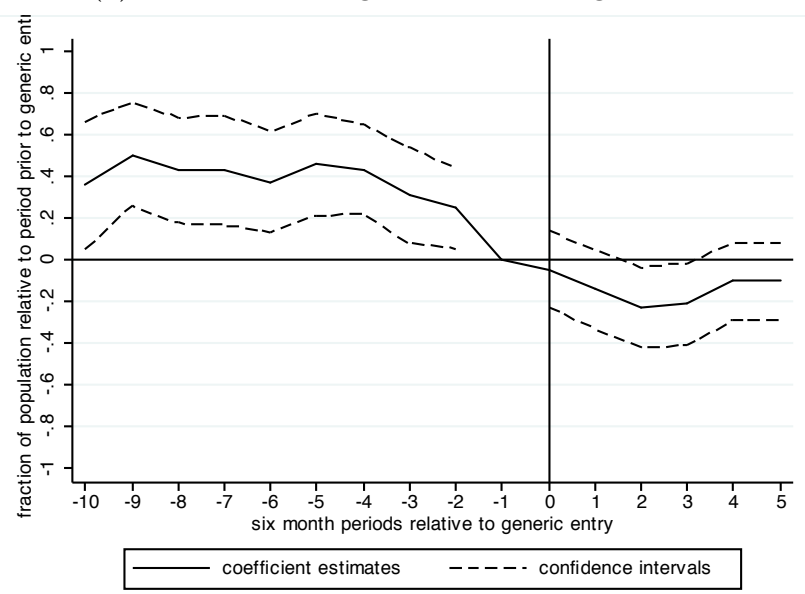

(c) Use of branded reformulation, ages 0-40

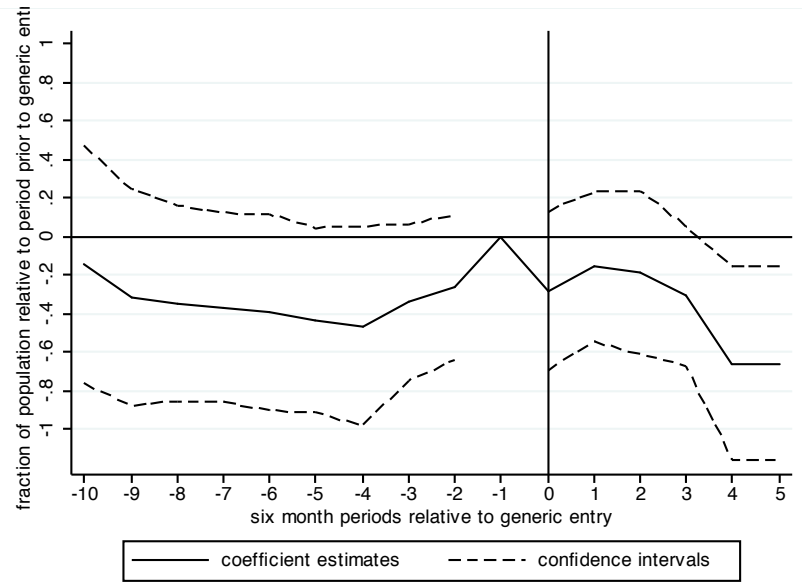

(b) Total use of original molecule, ages 40-80

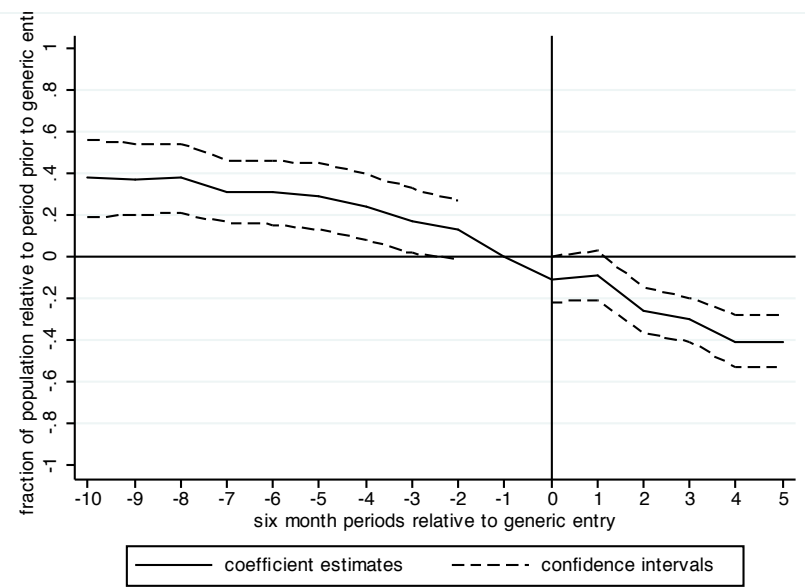

(d) Use of branded reformulation, ages 40-80

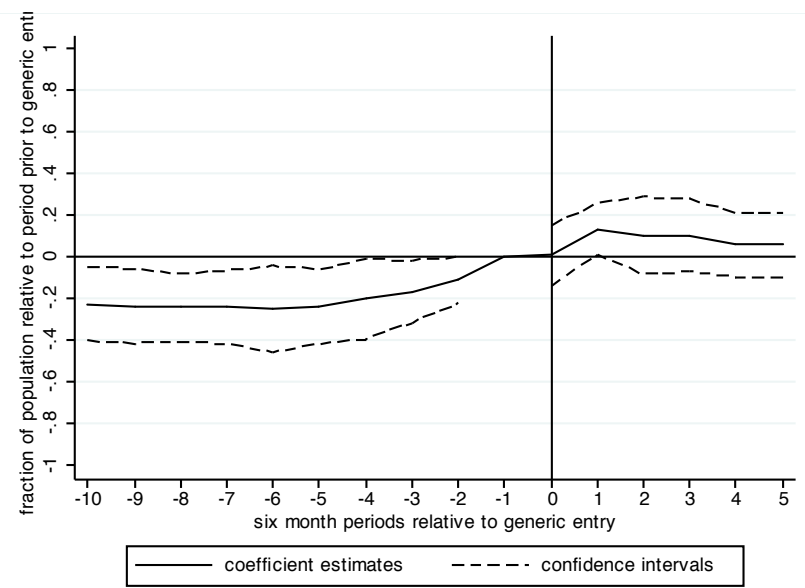

Notes: Solid lines are coefficient estimates, dashed lines are 95 percent confidence intervals. Standard errors clustered at the therapeutic class and six month period. Vertical line indicates generic entry. 
Figure 8: Original molecule overall use specification checks

(a) Omitting molecule-specific linear time trends

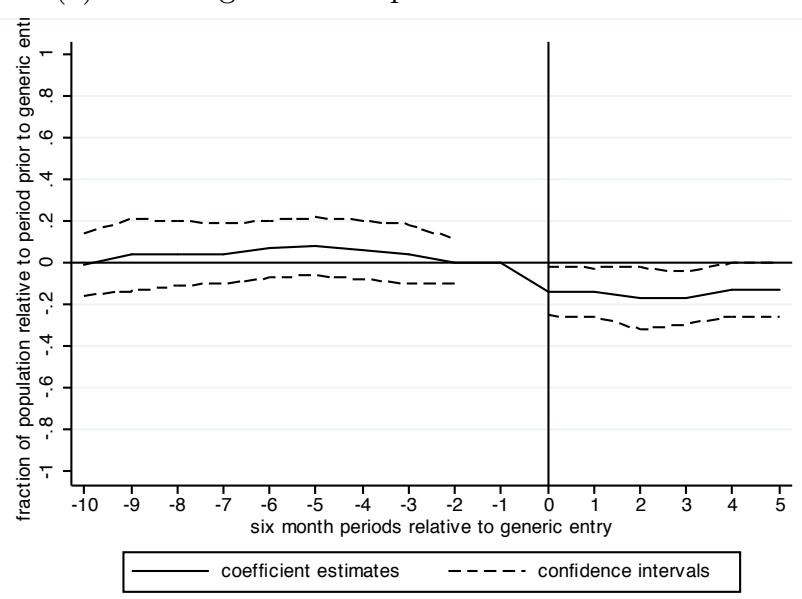

(c) Balanced panel sample

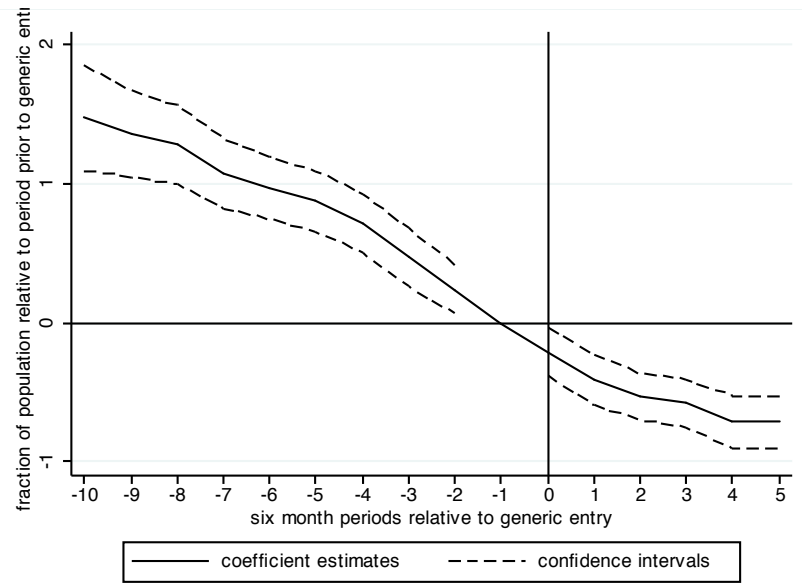

(b) Including class-time fixed effects

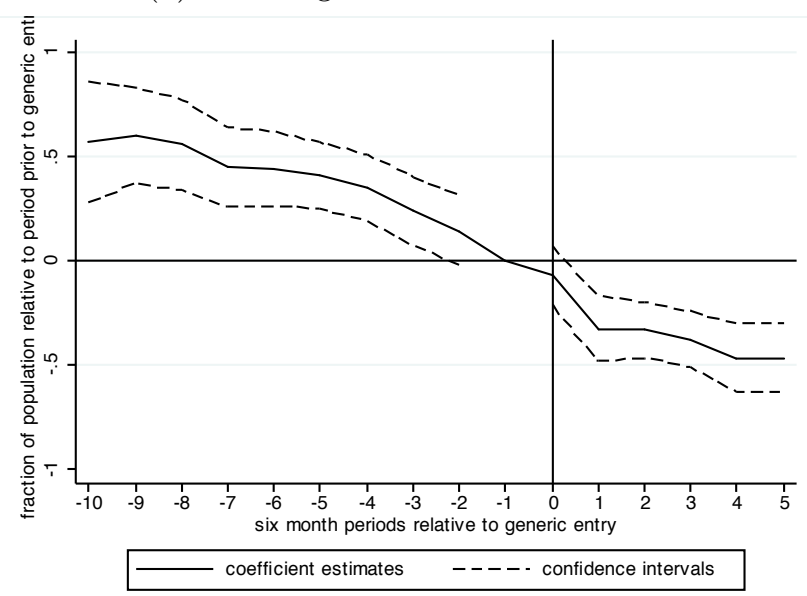

(d) No regression weights

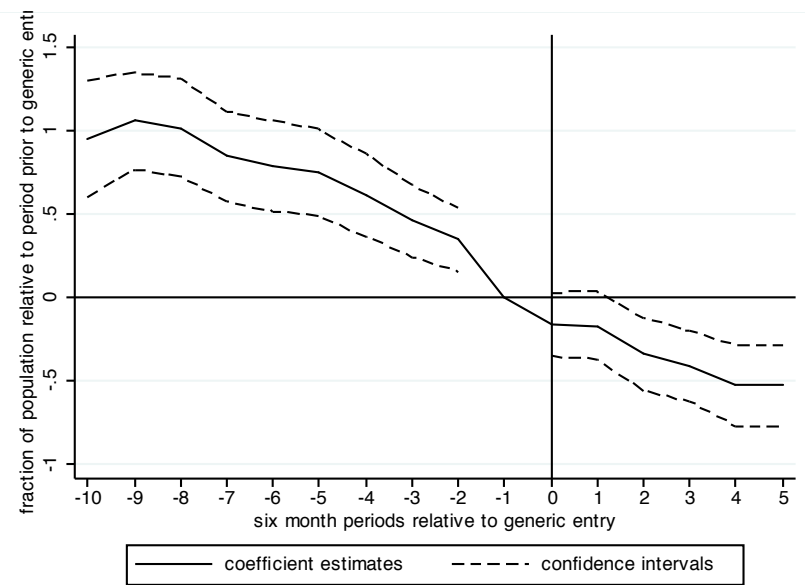

Notes: Solid lines are coefficient estimates, dashed lines are 95 percent confidence intervals. Standard errors clustered at the therapeutic class and six month period. Vertical line indicates generic entry. 
Figure 9: Reformulation use specification checks

(a) Omitting molecule-specific linear time trends

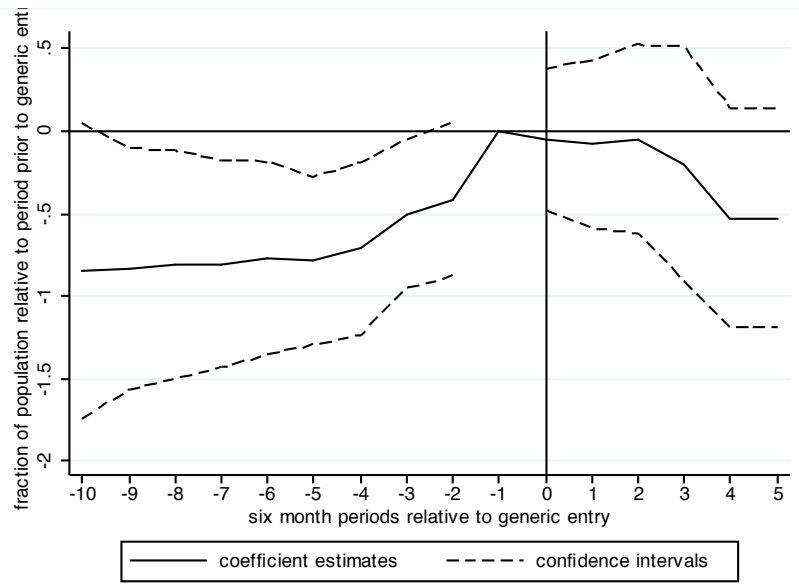

(c) No regression weights

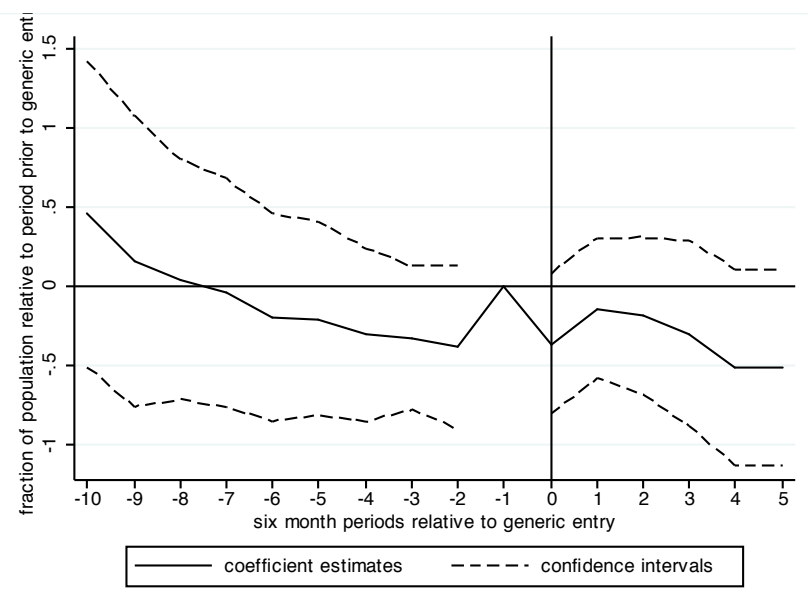

(b) Including class-time fixed effects

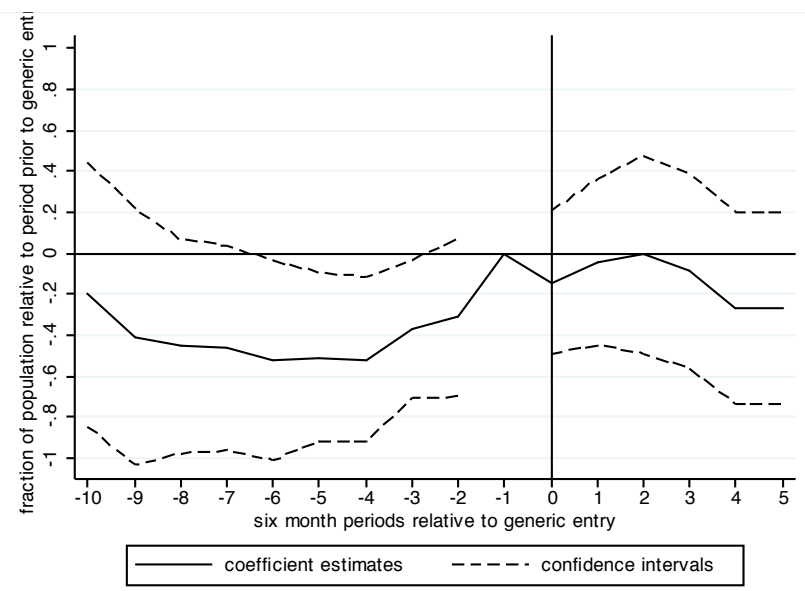

Notes: Solid lines are coefficient estimates, dashed lines are 95 percent confidence intervals. Standard errors clustered at the therapeutic class and six month period. Vertical line indicates generic entry. 
Figure 10: Metformin

(a) Average annual "lower bound" price for metformin

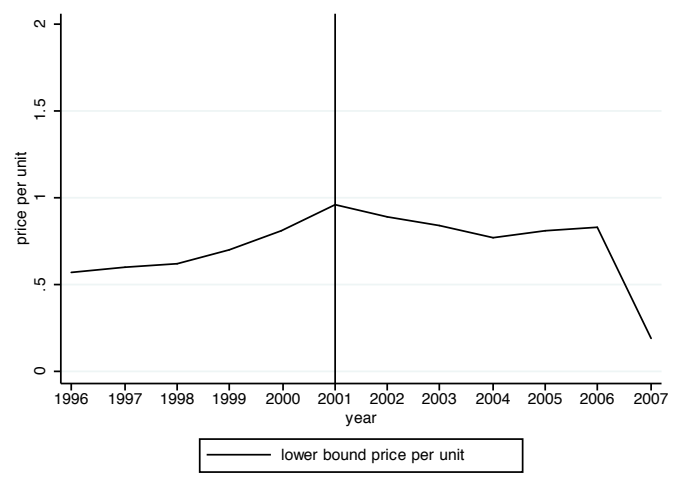

(c) Use of branded metformin and branded reformulations

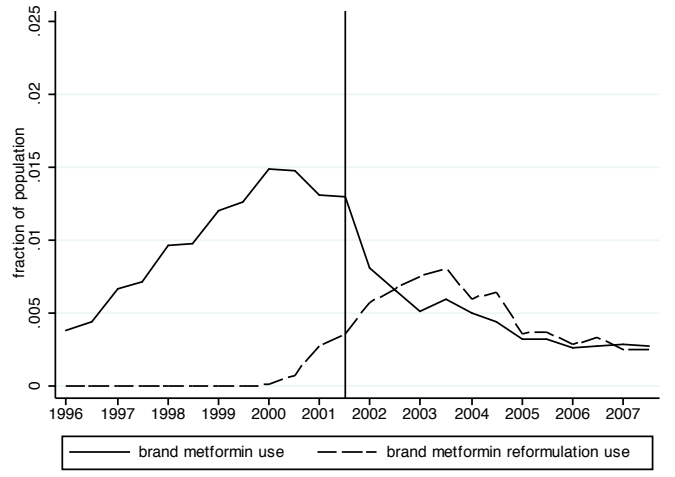

(e) Quarterly numbers of samples

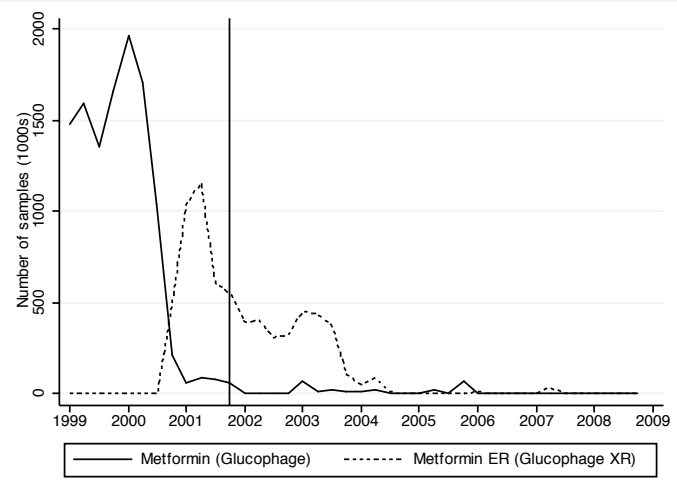

(b) Overall, brand, and generic utilization of metformin

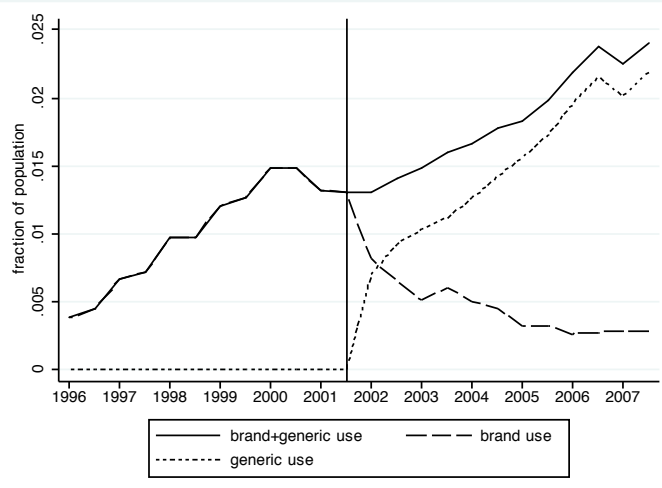

(d) Quarterly costs of detailing

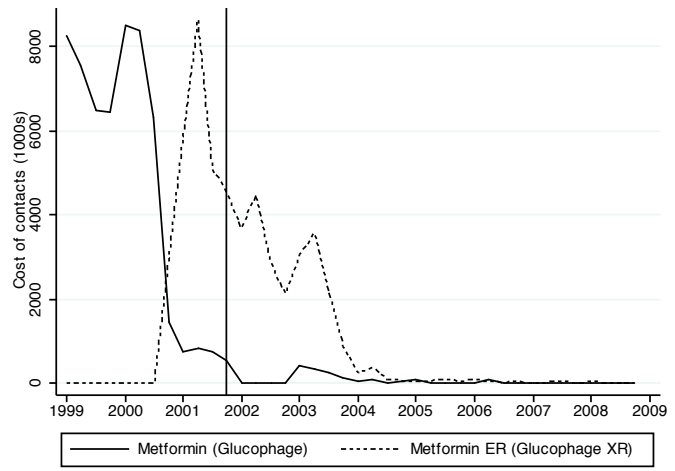

(f) Quarterly costs of advertising in medical journals

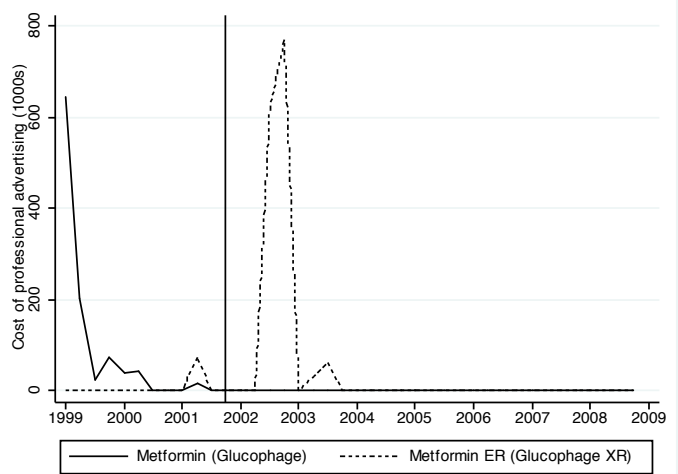

Notes: Vertical line indicates period (year for price, biannual for use, quarter for advertising) prior to generic entry for regular metformin in January 2002 . Price and utilization measures constructed from the MEPS. Source of advertising data: IMS Health, IMS Promotion Audits, $1 / 1999-12 / 2008$. 
Figure 11: Simvastatin

(a) Annual average "lower bound" price for simvastatin

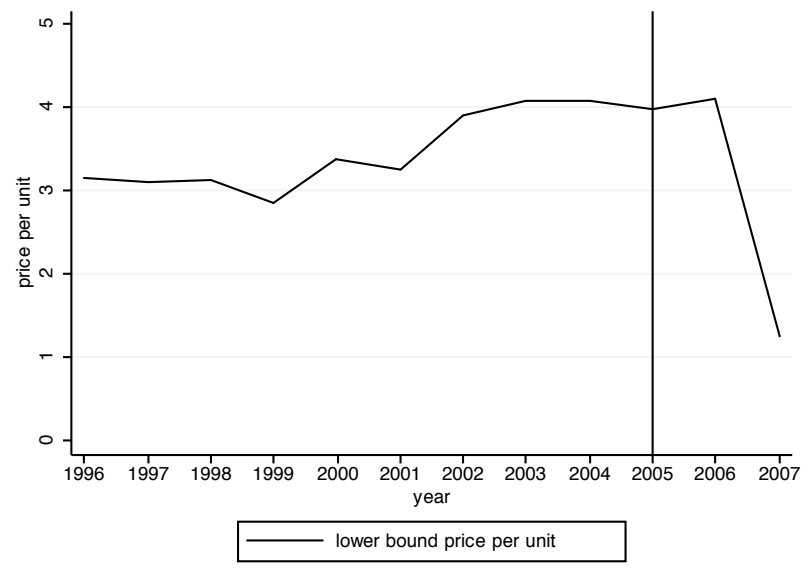

(c) Use of branded simvastatin and branded reformulation (simvastatin+ezetimibe)

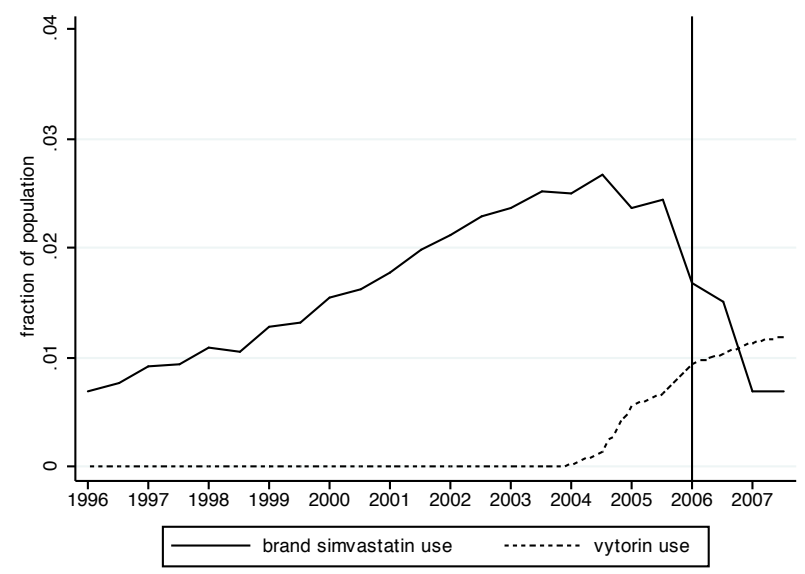

(e) Quarterly numbers of samples

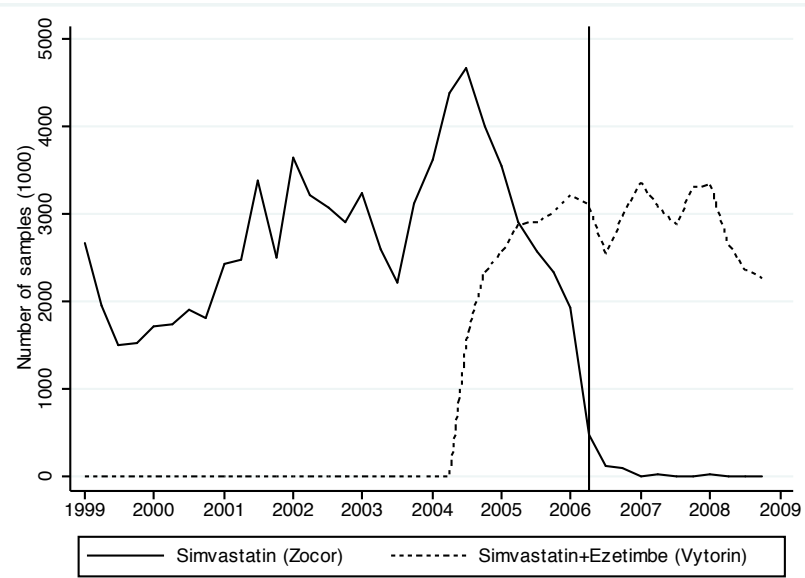

(b) Overall, brand, and generic utilization of simvastatin

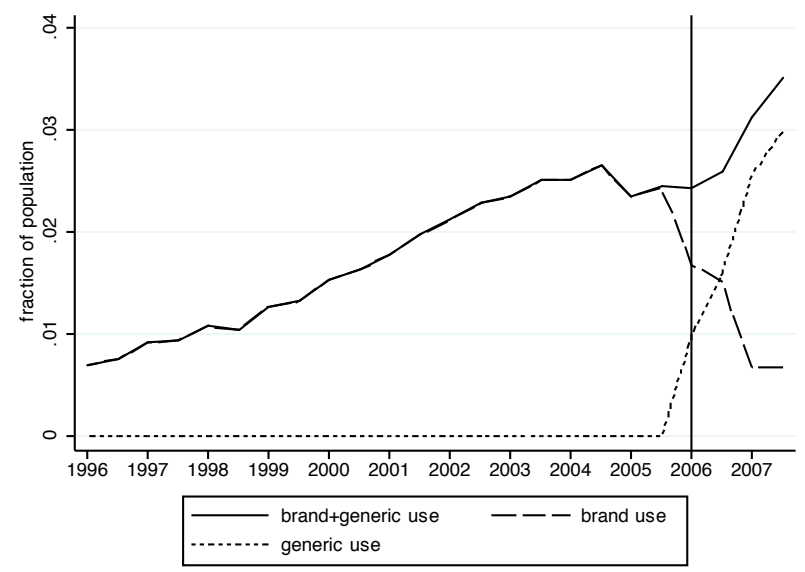

(d) Quarterly costs of detailing

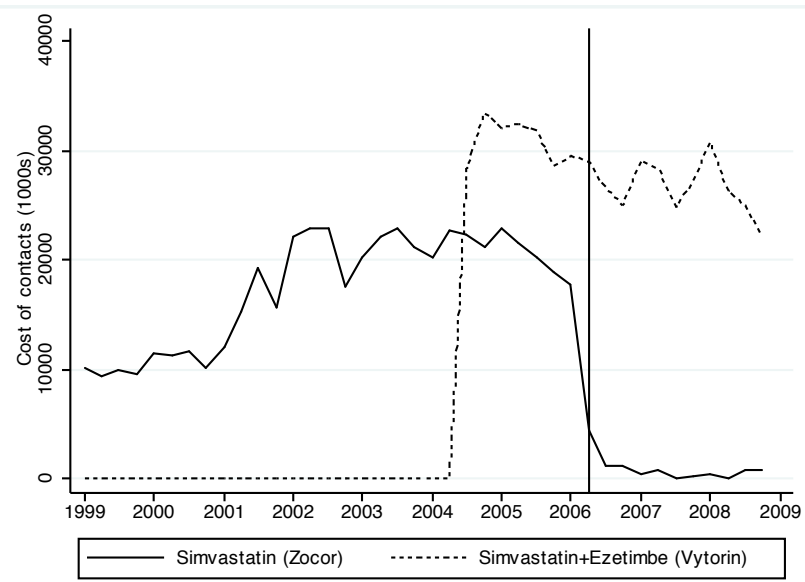

(f) Quarterly costs of advertising in medical journals

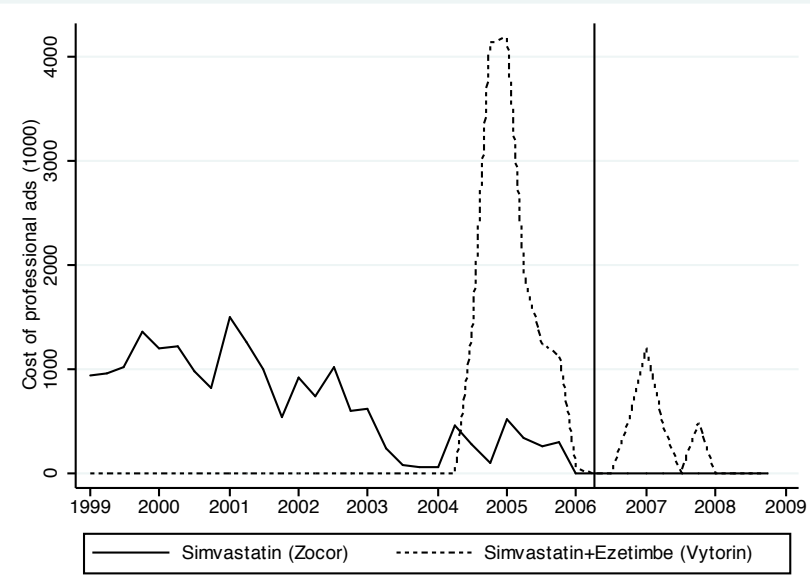

Notes: Vertical line indicates period (year for price, biannual for use, quarter for advertising) prior to generic entry for simvastatin in June 2006. Price and utilization measures constructed from the MEPS. Source of advertising data: IMS Health, IMS Promotion Audits, 1/1999-12/2008. 


\section{Figure 12: Bupropion SR and XL}

(a) Annual average "lower bound" price for bupropion SR

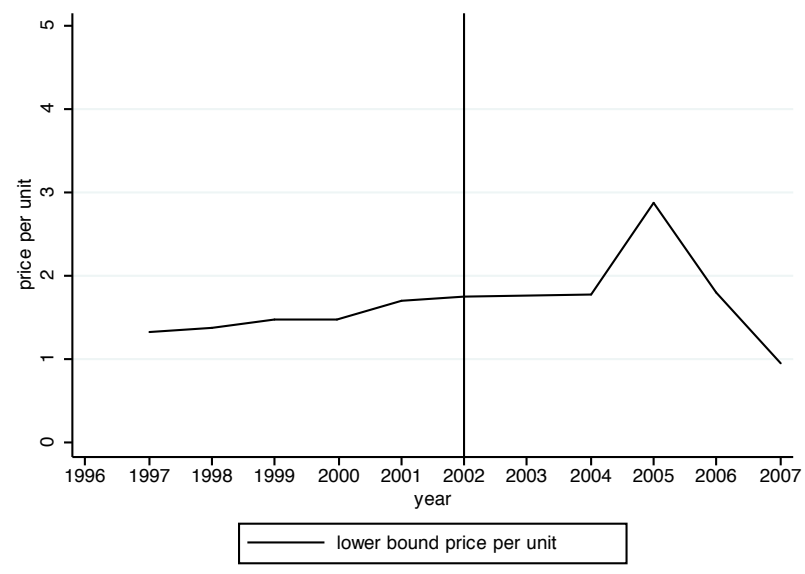

(c) Utilization of bupropion SR and XL

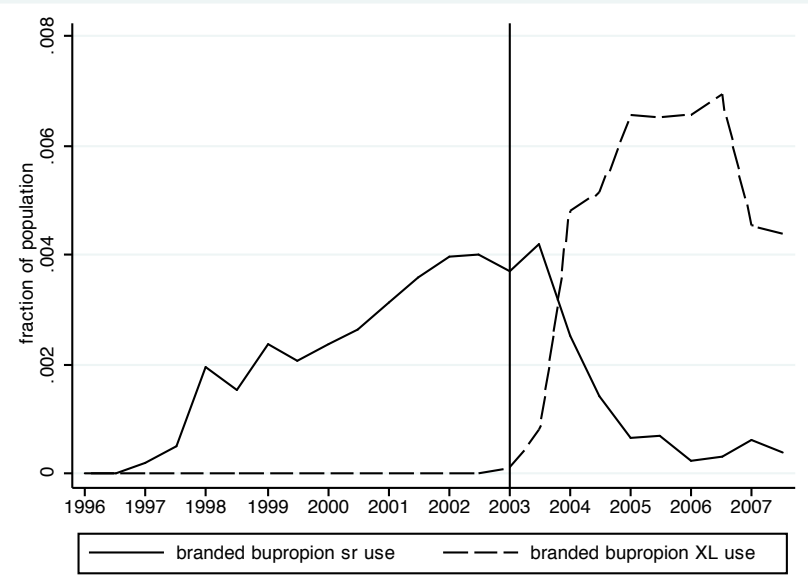

(e) Quarterly numbers of samples

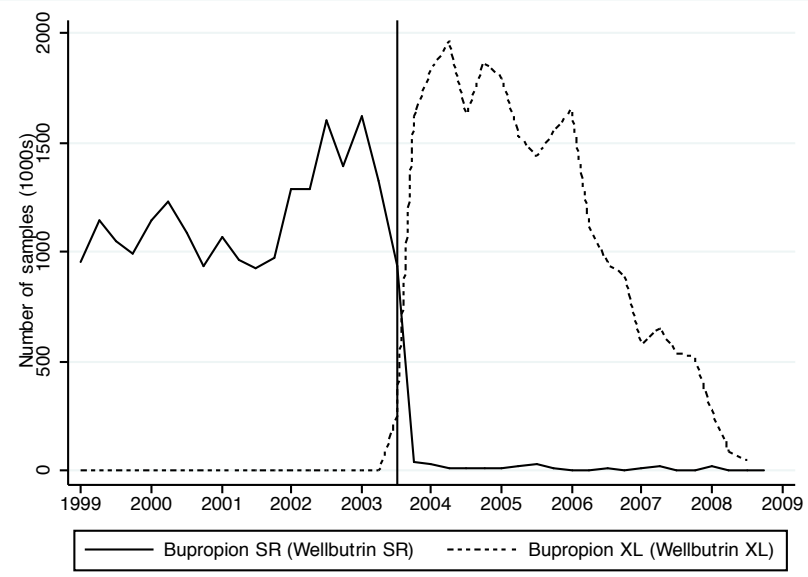

(b) Overall, brand, and generic utilization of bupropion SR

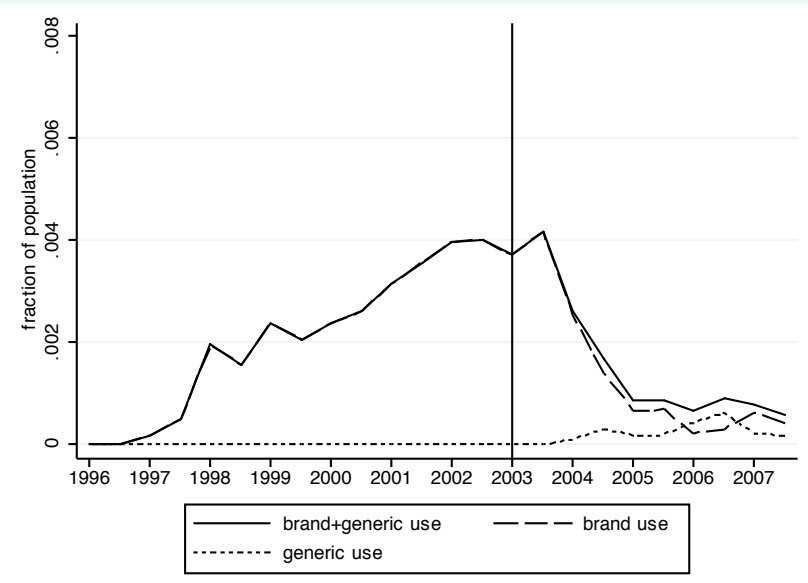

(d) Quarterly costs of detailing

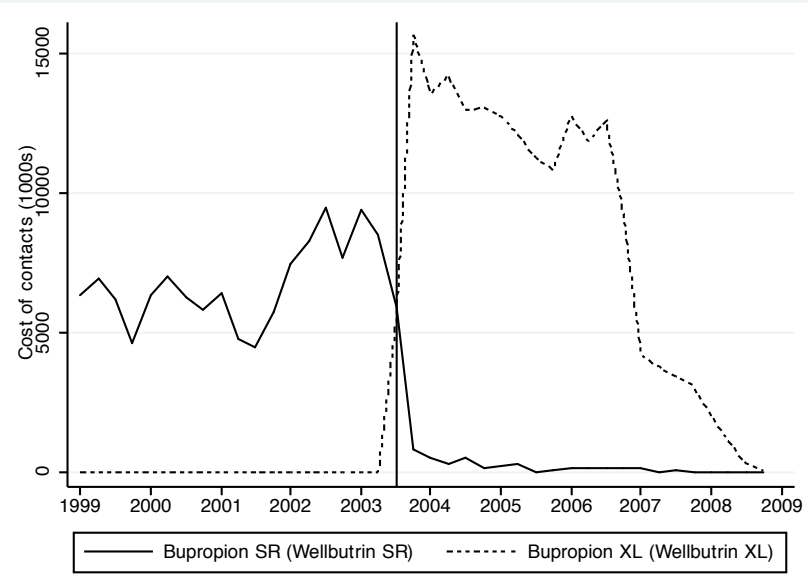

(f) Quarterly costs of advertising in medical journals

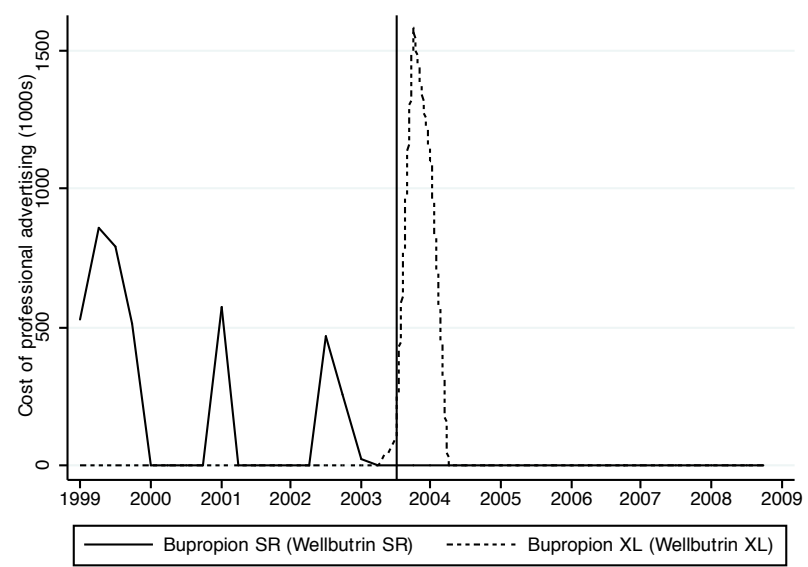

Notes: Vertical line indicates period (year for price, biannual for use, quarter for advertising) prior to generic entry for buproprion SR in November 2003. Price and utilization measures constructed from the MEPS. Source of advertising data: IMS Health, IMS Promotion Audits, 1/1999-12/2008. 
Figure 13: Citalopram

(a) Average "lower bound" price for citalopram

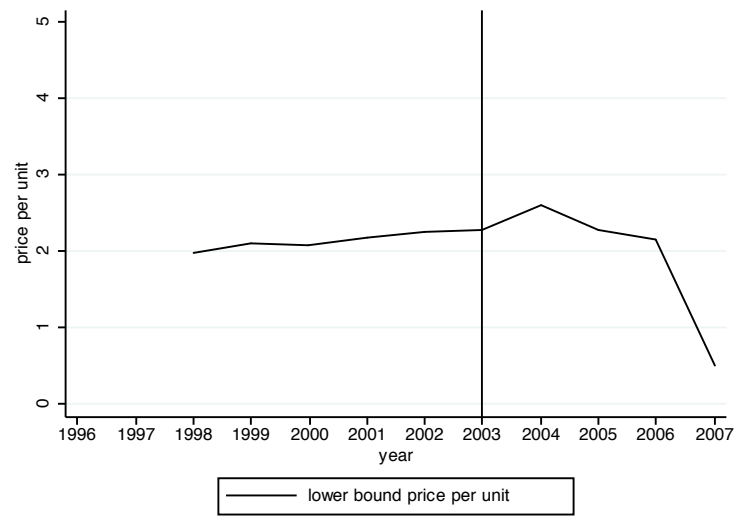

(c) Use of branded citalopram and branded escitalopram

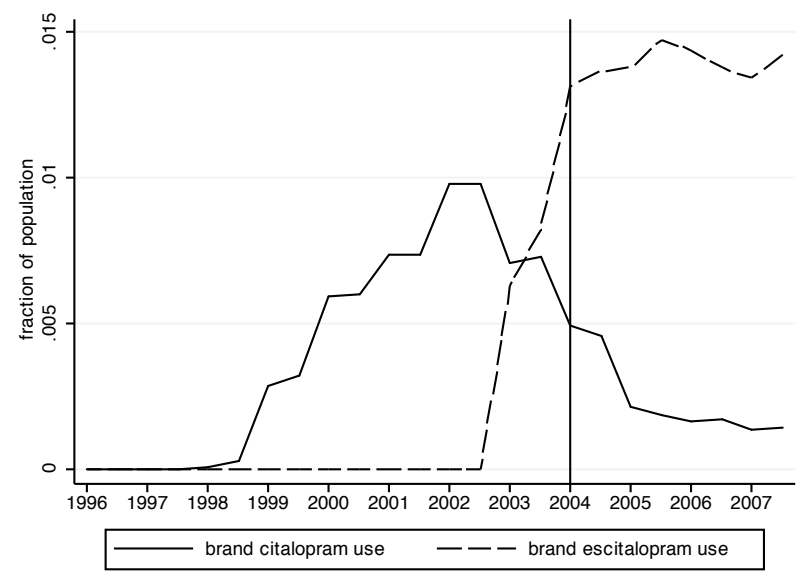

(e) Numbers of samples

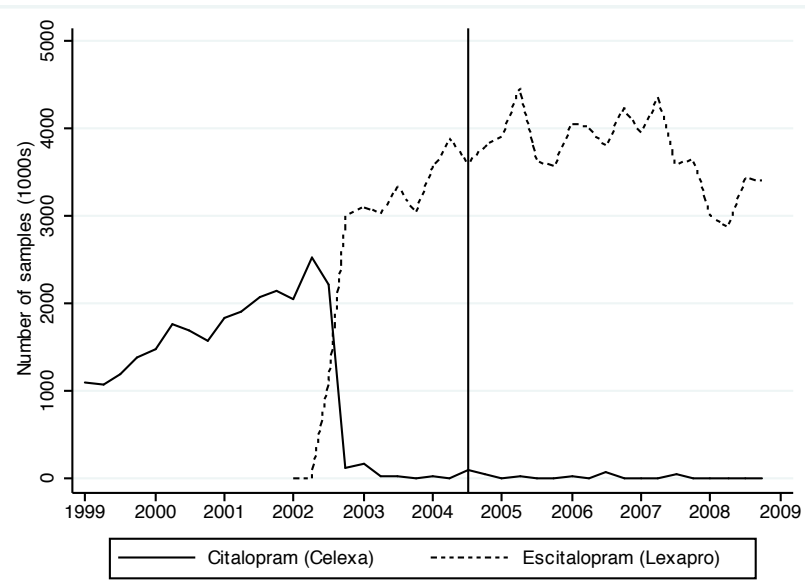

(b) Overall, brand, and generic utilization of citalopram

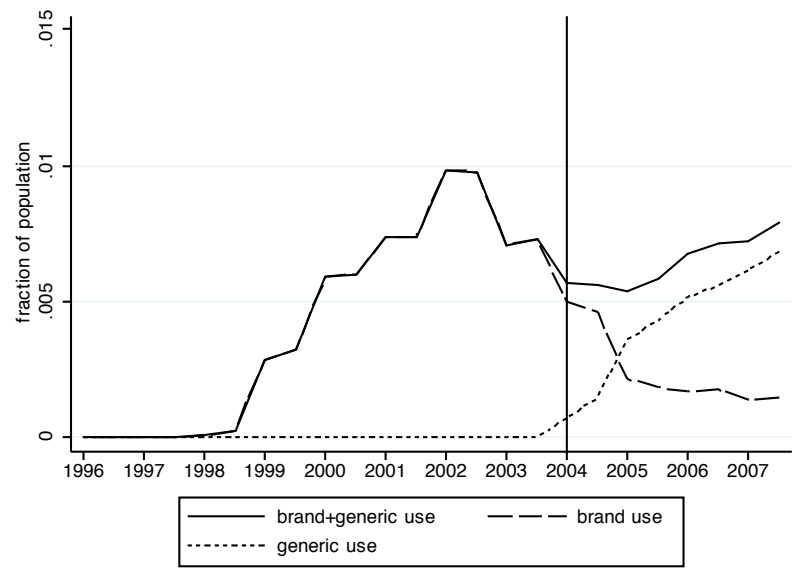

(d) Costs of detailing

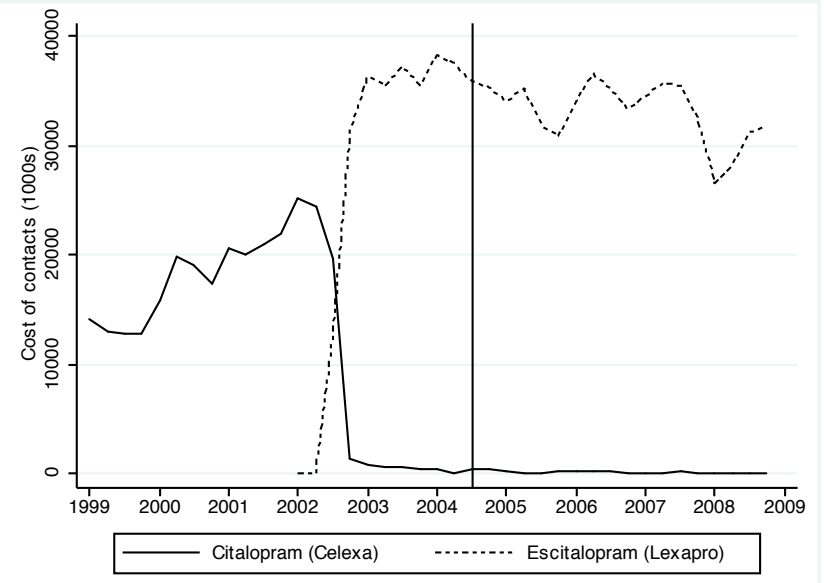

(f) Total promotion costs

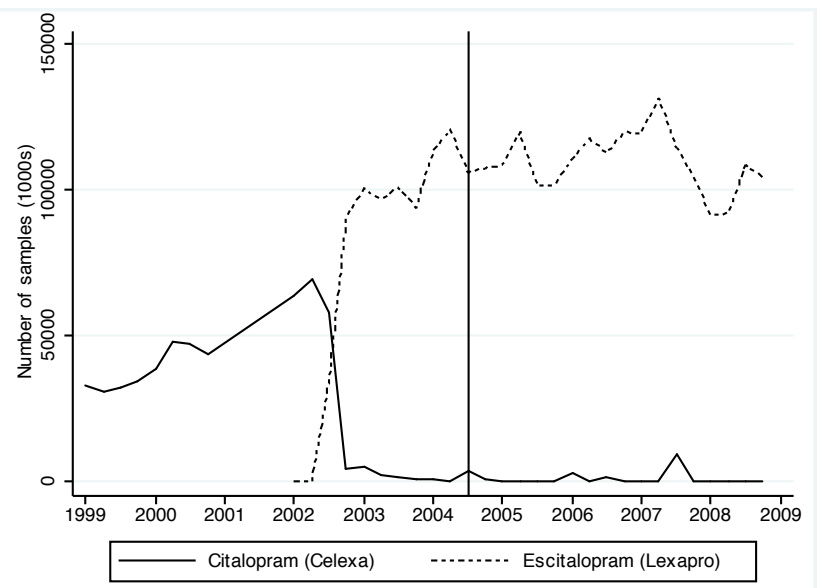

Notes: Vertical line indicates period (year for price, biannual for use, quarter for advertising) prior to generic entry for citalopram in October 2004. Price and utilization measures constructed from the MEPS. Source of advertising data: IMS Health, IMS Promotion Audits, 1/1999-12/2008. Missing total promotion data for 2001. 


\section{A Data Construction}

The main source of data for the analysis is the Medical Expenditure Panel Survey from the Agency for Healthcare Research and Quality. The MEPS consists of 11 overlapping two-year interview panels. Each panel contains a nationally representative sample of around 16 thousand individuals who are interviewed over the five interview rounds across the two years. We calculate prices and utilization probabilities using the MEPS Full Year Consolidated component files and the Prescribed Medicines Component Files. The Full Year Consolidated file includes demographic and labor market information, sample weights, and a rich set of round-specific health insurance coverage information. The Prescribed Medicines component collects information from the actual pharmacies where survey participants obtain their prescriptions. The information obtained from pharmacies includes the national drug code and name of the drug, the strength and quantity obtained, the total price, as well as the amounts paid by different insurance sources and the patient. The MEPS is able to match only 50 percent of survey respondents prescription mentions to pharmacy information. The MEPS imputes the information for non-matched drug mentions (Moeller et al. 2001). We only use the matched prescriptions for our average price calculations, but we use all prescription mentions to calculate utilization probabilities. Presumably, the imputations for the brand or generic status of a drug is simpler and more accurate than imputed detailed price and insurance payer information.

The first step for estimating utilization probabilities is to create patient-level indicator variables for obtaining each drug molecule in a particular round. The Prescribed Medicines Component includes the national drug code and the pharmacist provided drug name for each prescription mention. The drug code is often missing, so we use the drug name to identify utilization. There are occasional mistakes in drug names, such as a generic version of a drug molecule being obtained before the FDA approved the generic version (i.e. fluoxetine versus Prozac). We expect that this reflects the substitution of the molecule name for the brand name. Prior to generic entry we treat these mentions as brand reports, but we are unable to distinguish between correct and incorrect generic or brand reports following generic entry. Next, we convert the person-round drug indicator variables to person-biannum drug indicator variables. While we do not know the exact date a person obtains a drug, we know the start and end dates of each round for each person in the sample. We assume that if an individual obtains a drug in a given round, they use the drug for the entire round. Then, the person-biannum drug indicator is set to on for any biannual period in which the round occurs. We treat the resulting person-biannum data set as repeated biannual 
cross-sections. Using the sample weights, we calculate the fraction of the population obtaining each drug molecule in each biannum. The specific utilization outcomes we construct are the probability of obtaining the branded version of a molecule in a given biannum, the probability of obtaining the generic version of a molecule, the brand market share for each molecule, the overall probability of obtaining a molecule (brand or generic), and the probability of obtaining a branded reformulation of a molecule facing generic competition.

We are also interested in how changes in prescription drug utilization vary across individuals with different prescription benefit designs. Specifically, we may expect that beneficiaries with plans that place more restrictions on obtaining branded drugs (such as HMOs or Medicaid) may be less likely to switch to a new branded molecule following generic entry than patients with less restrictive fee-for-service or PPO plans. Thus, we estimate utilization probabilities separately for individuals with private HMO health plans, employer provided health insurance that is not from a HMO, Medicaid recipients, and individuals aged 65 or older. We use monthly employer provided health insurance and Medicaid variables in the MEPS Full Year Consolidated Files to determine whether an individual had employer provided health insurance or Medicaid ever in a six-month period. There are round-specific HMO variables from the Person Round Plan component that indicate whether an individual had employer provided HMO coverage in each round. Thus, the non-HMO employer coverage is defined as having employer coverage in a biannual period, but having no HMO coverage during rounds that occurred during that biannual period. It should be noted that individuals select into different types of health insurance. This non-random selection, as well as differences in prescription benefit design, will drive differences in utilization patterns across health insurance types.

We also construct price outcome variables using the MEPS Prescribed Medicines component. We calculate average prices only using prescription mentions that are matched to pharmacist reports. We create a person-round data set that gives the average unit price (across dosages) paid by each person who obtained a prescription in a given round. We convert the person-round file to a person-biannum file by taking weighted averages of the person-round averages, with the weights depending on the amount of a round that occurred in a given biannum. We use the person-biannum data and sample weights to create average unit prices for each molecule in each biannum. For the empirical work, we convert the nominal unit prices to real prices using year 2000 dollars, and then convert prices to natural log units. Average out-of-pocket prices are zero for some observations. 
Thus, for all out-of-pocket and total price outcomes (in order to be consistent in each case), we add one before taking the natural log.

Prices in the case study graphs are calculated annually and do not drop imputations. We did this in part to compare aggregate expenditures with MEPS-generated aggregates. These prices are calculated as total expenditures in each year divided by total units (i.e. pills) sold in each year. These averages are slightly different, as they provide the average price of a prescription of a given drug, as opposed to the average of within-person average prices, which is used in our price regressions. Individuals with more prescriptions may find cheaper prices, and will receive higher weighting in the latter calculation. However, the average unit prices and patterns are qualitatively calculated either way.

The specific price outcomes we construct include total branded price, out-of-pocket branded price, total average price (across brand and generic molecules), average out-of-pocket price, and finally total and out-of-pocket "lower-bound" prices. The lower bound price is equal to the average branded price prior to generic entry and the average generic price following. We only observe individuals selecting into branded and generic drugs. It may be that individuals who chose generic drugs face higher brand prices, and individuals who choose branded drugs face lower co-payments for generic drugs. Thus, our branded drug, and our "lower-bound" prices may not reflect the actual average total and out-of-pocket brand and generic prices facing beneficiaries. However, the average prices should accurately reflect the average price faced by individuals acquiring these drugs (aside from selection issues stemming from the non-matched individuals in the Prescribed Medicines component).

We have generated total expenditures and total numbers of prescriptions to compare with AHRQ- constructed aggregates for highly used drugs. We are typically within 1 to 2 percent of the MEPS aggregates, these comparisons are available upon request. 


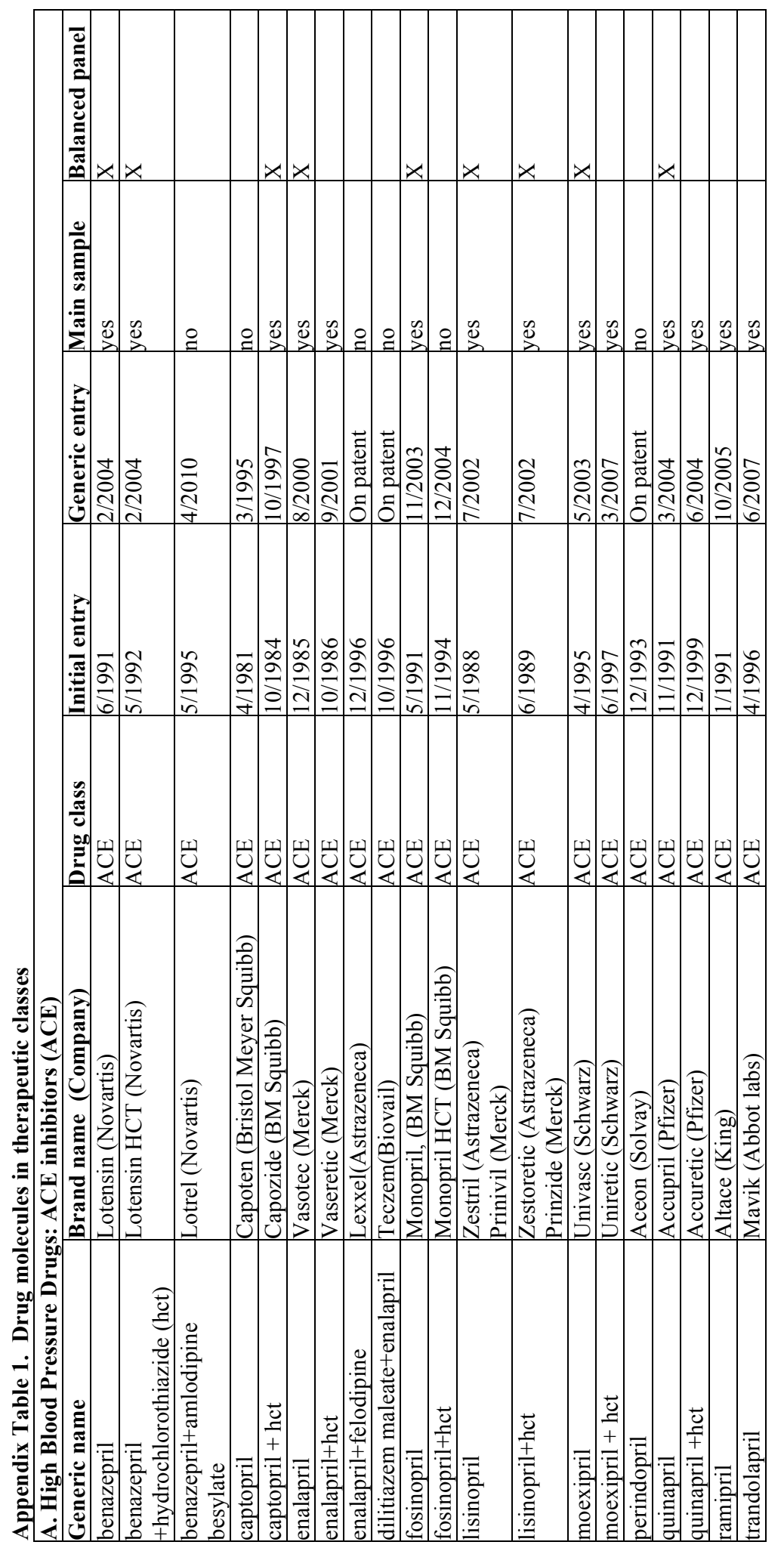




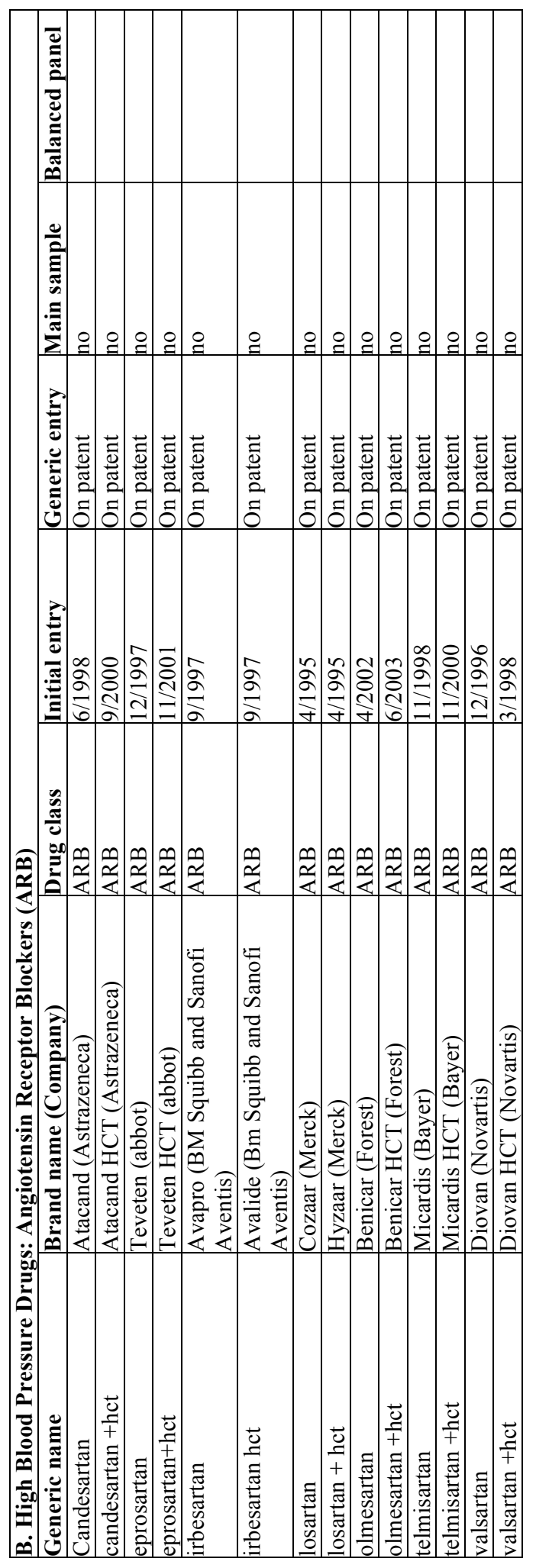




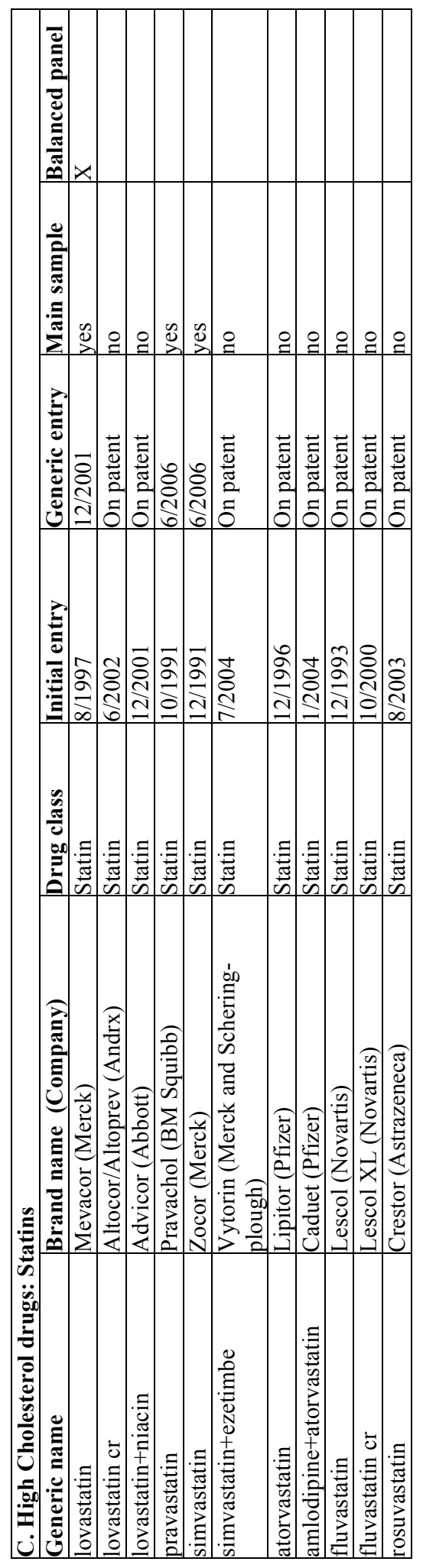




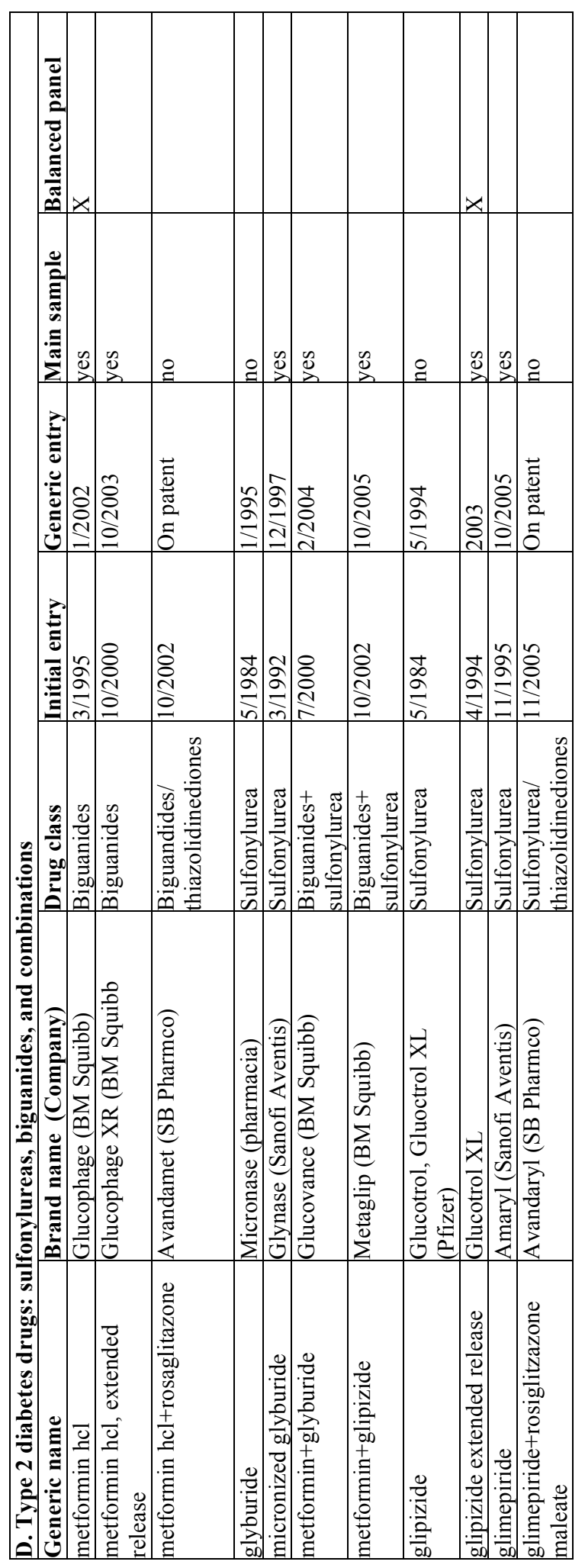




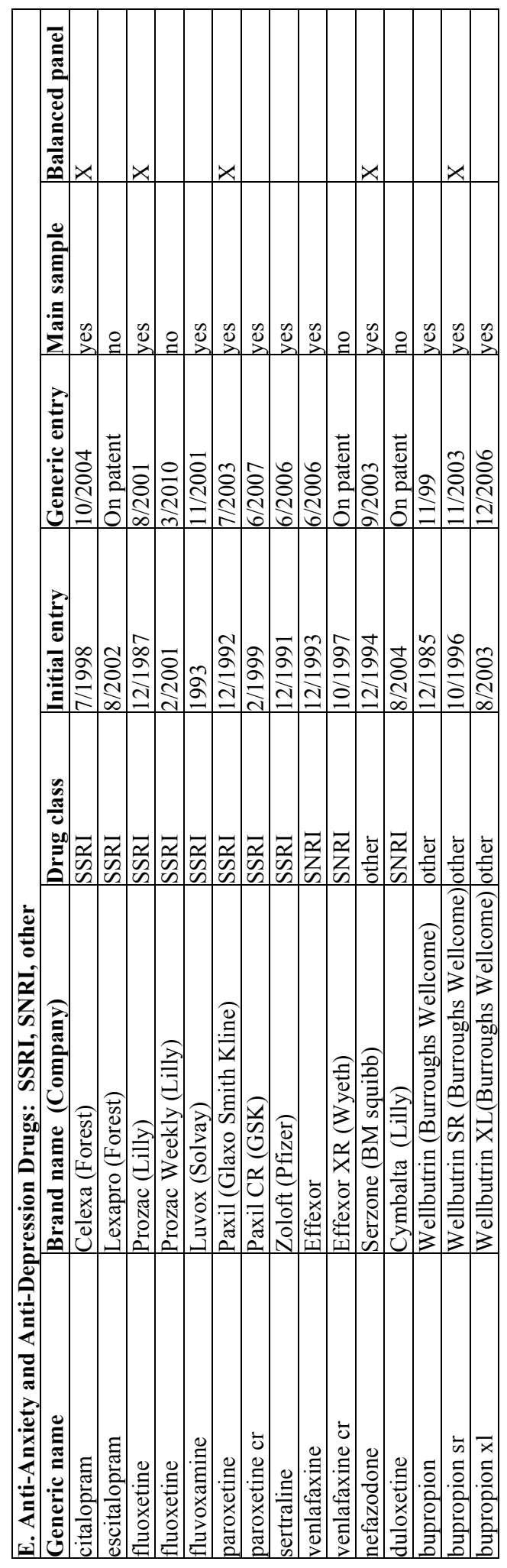




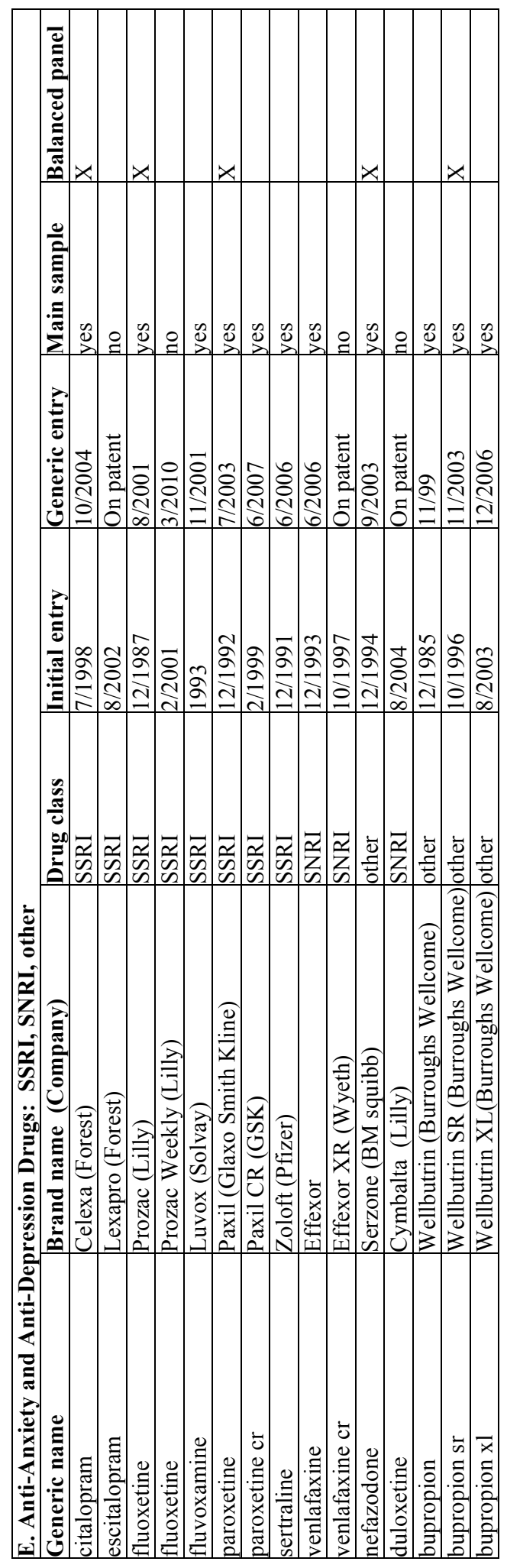




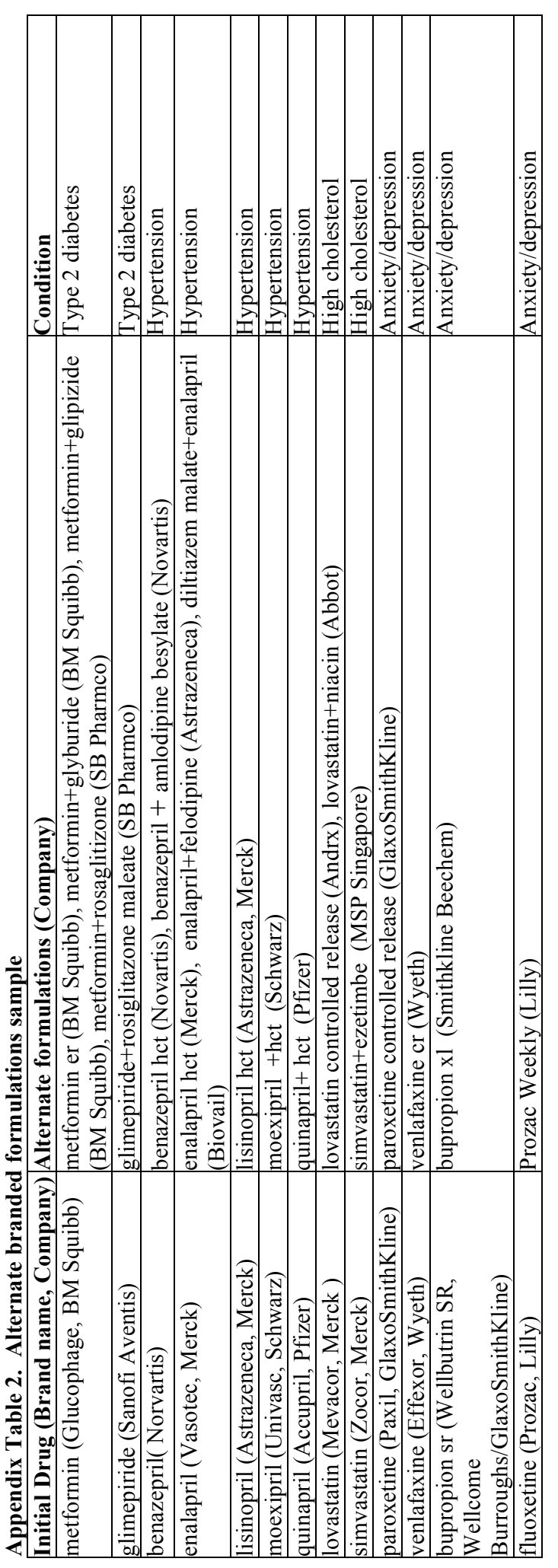

